\title{
EMPLEO FORMAL Y LIBERTAD ECONÓMICA REGIONAL EN MÉXICO
}

\author{
FORMAL EMPLOYMENT AND REGIONAL ECONOMIC FREEDOM IN MEXICO
}

\section{EMPREGO FORMAL E LIBERDADE ECONÔMICA REGIONAL NO MÉXICO}

\author{
Isaac Sánchez Juárez ${ }^{1}$ \\ Rosa M. García-Almada²
}

\begin{abstract}
Resumen
¿La libertad económica se correlacionó positivamente con el empleo formal en México? La investigación que se presenta responde a esta pregunta. Se usaron datos del periodo 2003-2015 de las treinta y dos entidades federativas, así como de las regiones frontera norte y sur, a partir de lo cual se crearon varios paneles estimados con los métodos de efectos fijos, aleatorios y generalizado de momentos (GMM). Usando GMM para las treinta y dos entidades se encontró que la libertad económica se correlacionó negativamente con el empleo, la elasticidad fue -0.04. Para la frontera norte se halló que la elasticidad del empleo con respecto a la libertad económica fue positiva (aunque estadísticamente no significativa), mientras que en la región sur la elasticidad fue negativa (-2.20). Por ello, reducir el gasto del gobierno y los impuestos, así como flexibilizar el mercado de trabajo (mayor libertad económica) no son las mejores opciones de política para crear empleo formal.
\end{abstract}

Palabras claves: México; frontera norte; región sur; libertad económica; empleo.

Doi: https://doi.org/10.15359/eys.25-57.7

Recibido: 12-02-2020. Reenvíos: 24-02-2020, 13-04-2020, 16-04-2020, 21-04-2020, 27-04-2020. Aceptado: 27-042020. Publicado: 01-05-2020.

${ }^{1}$ Universidad Autónoma de Ciudad Juárez, México. Doctorado en Estudios Regionales por El Colegio de la Frontera Norte. Miembro del Sistema Nacional de Investigadores de México. Correo electrónico: isaac.sanchez@uacj.mx ORCID: http://orcid.org/0000-0002-1975-5185

2 Universidad Autónoma de Ciudad Juárez, México. Doctorado en Estudios Regionales por El Colegio de la Frontera Norte, México. Miembro del Sistema Nacional de Investigadores de México. Correo electrónico: maria.garcia@uacj.mx ORCID: https://orcid.org/0000-0003-2330-8385

Isaac Sánchez Juárez y Rosa M. García-Almada

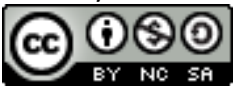

Revista Economía y Sociedad by Universidad Nacional is licensed under a CreativeCommons Reconocimiento-NoComercial- 


\begin{abstract}
The paper aimed to answer whether economic freedom positively correlated with formal employment in Mexico. Data for the 2003-2015 period from the thirty-two federal entities and the northern and southern borders were used to create estimated panel data through fixed effects, random effects, and generalized method of moments (GMM). Economic freedom was negatively correlated with employment and elasticity was 0.04 for the thirty-two entities, while the elasticity of employment was positive (although statistically not significant) with respect to economic freedom for the northern border but negative $(-2.20)$ for the southern region. Consequently, reducing government expenditure and taxes, as well as making the job market more flexible (greater economic freedom) are not the best policy options to create formal employment.
\end{abstract}

Keywords: Mexico; northern border; southern region; economic freedom; employment

\title{
Resumo
}

A liberdade econômica correlacionou positivamente com o emprego formal no México? A pesquisa apresentada responde à pergunta. Dados do período 2003-2015 foram utilizados nas trinta e duas unidades federativas, bem como nas regiões da fronteira norte e sul, a partir dos quais vários painéis avaliados foram criados mediante os métodos de efeitos fixos, aleatórios e dos momentos generalizados (GMM). Ao usar o GMM para as trinta e duas entidades, verificou-se que a liberdade econômica foi correlacionada negativamente com o emprego, sendo a elasticidade de $-0,04$. Para a fronteira norte, verificou-se que a elasticidade do emprego em relação à liberdade econômica era positiva (embora não estatisticamente significativa), enquanto na região sul a elasticidade era negativa $(-2,20)$. Portanto, reduzir os gastos do governo e os impostos, e tornar o mercado de trabalho mais flexível (maior liberdade econômica) não são as melhores opções políticas para a criação de emprego formal.

Palavras-chave: México; fronteira norte; região sul; liberdade econômica; emprego. 


\section{Introducción ${ }^{3}$}

La economía mexicana no ha sido capaz de generar el volumen suficiente de empleos formales para incorporar a todos aquellos individuos que periódicamente buscan ser parte del mercado de trabajo. Desde un enfoque ortodoxo, la explicación radica en un bajo nivel de libertad económica (LE) que impide mejore la inversión productiva y la productividad total de los factores, por lo cual la producción de bienes y servicios no crece como debería para generar el empleo requerido.

En virtud de esto, en el caso mexicano se implementó un modelo económico cuya meta principal era aumentar la LE para, en teoría, contribuir al crecimiento económico y empleo. En este artículo de investigación se verifica econométricamente la relación entre las variables mencionadas para lo cual se utilizaron datos a nivel de entidad federativa del 2003-2015 (el periodo de tiempo estuvo determinado por la disponibilidad de la información). Como variables de control se recurrió a la producción no manufacturera, producción manufacturera y la crisis financiera internacional del 2008-2009.

El documento está dividido en tres partes. En la primera se expone el marco de referencia y la bibliografía revisada para proporcionar un punto de comparación que permita resaltar la originalidad del presente esfuerzo. En la segunda se presenta, con fines de replicabilidad, la fuente de los datos, así como el tratamiento de estos y los procedimientos econométricos aplicados. En la tercera parte se describen las series y se plantean los resultados para, finalmente, concluir y discutir algunos elementos de política económica.

\section{Empleo y libertad económica: Marco de referencia y revisión bibliográfica}

Dado que no existe un marco formal que dé cuenta de la relación entre la LE y el empleo, se presume que este último está determinado positivamente por la producción de bienes y servicios. A su vez, se supone que el crecimiento de la producción de bienes y servicios depende positivamente de la productividad total de los factores y la inversión, mientras que estas son determinadas por la LE. Por tanto, si los aumentos en LE contribuyen al aumento de la producción de bienes y servicios, permitirán crear empleo formal.

Si la economía tiene elevados niveles de LE, logrará reducir los costos y aumentar los beneficios de las posibles inversiones. Además, los incrementos en la LE se supone favorecen un incremento en la productividad, particularmente laboral, al promover la adquisición de mayores destrezas por parte de los grupos trabajadores, al considerar estos que lo que reciben (salarios) es mayor

\footnotetext{
${ }^{3}$ Los autores agradecen Sistema Nacional de Investigadores de Consejo Nacional de Ciencia y Tecnología en México por el financiamiento otorgado.
}

Isaac Sánchez Juárez y Rosa M. García-Almada

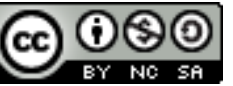

Revista Economía y Sociedad by Universidad Nacional is licensed under a CreativeCommons Reconocimiento-NoComercial- 
que sus costos (sacrificios); lo anterior produce crecimiento y este aumento del empleo formal (ver Figura 1).

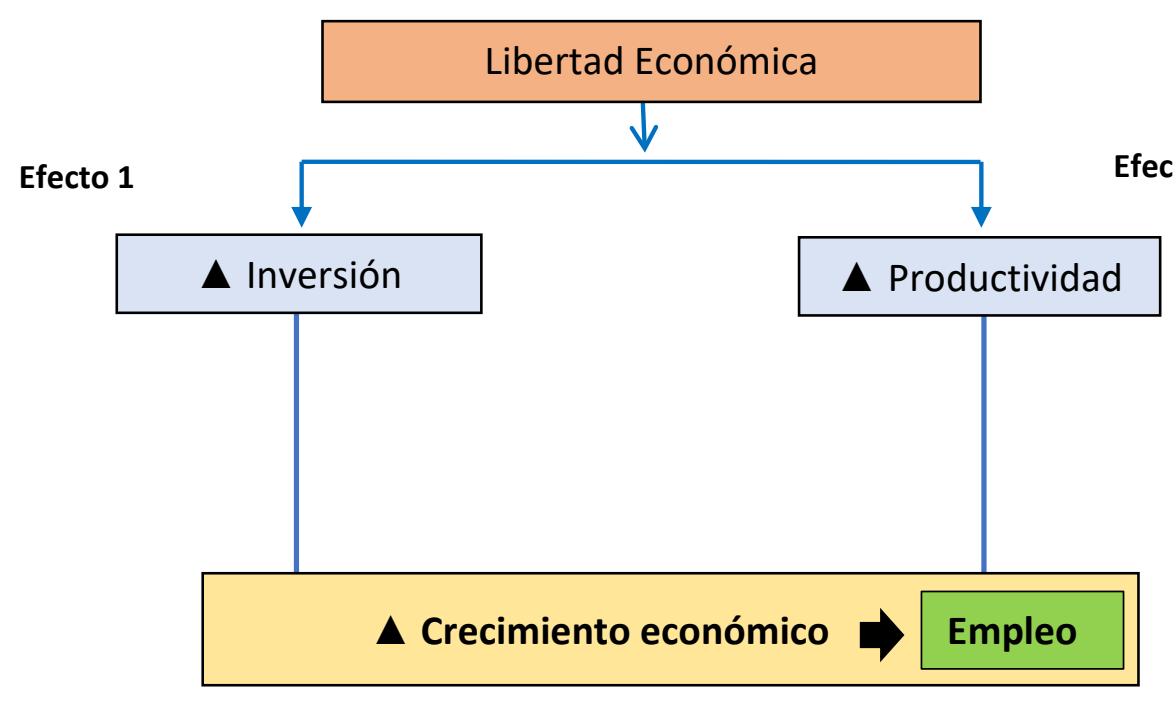

Figura 1. Libertad económica y crecimiento. Fuente: Elaboración propia con base en Sánchez y García (2014, p. 62).

En lo que refiere a la parte empírica, se buscaron, en diferentes bases de datos, estudios que para el caso mexicano hubieran abordado la relación entre las variaciones del empleo formal y alguna medida de libertad económica, pero no se encontró ningún trabajo, principalmente porque hasta donde se sabe la base de datos sobre libertad económica regional que aquí se usó es poco conocida por los equipos de investigación. ${ }^{4}$ No obstante, se lograron identificar algunos trabajos para el caso mexicano que evaluaron el impacto de la LE (o alguna medida relacionada) sobre el crecimiento económico.

Uno de los primeros trabajos localizados es el de Sánchez (2009), quien expone las razones por las cuales se considera que la LE es un motor del crecimiento económico, usa como referencia autores económico-liberales como Adam Smith, Friedrich Hayek y Milton Friedman. En este trabajo se exponen evidencias internacionales de la relación existente entre LE y crecimiento económico, la mayor parte de las cuales muestran que existe una correlación positiva entre las mencionadas variables.

Sánchez (2009), usando un índice de LE construido por la Heritage Foundation, demostró que la LE en México está en un nivel moderado, aunque a lo largo del tiempo presenta una tendencia a

${ }^{4}$ Para una discusión respecto al concepto de libertad económica leer Gwartney y Lawson (2003). 
incrementarse, por lo que asume que la falta de crecimiento económico vigoroso puede explicarse por la baja tasa de crecimiento de la LE. No obstante, reconoce la existencia de una relación negativa entre la tasa de crecimiento de la LE y el PIB per cápita y una muy baja correlación entre las series para el periodo 1996-2006.

Sobre la base de la información disponible, Sánchez (2009) concluye que deben realizarse una serie de reformas estructurales para incrementar la eficiencia del sector público, desregular la actividad económica, eliminar reglamentaciones excesivas, flexibilizar el mercado laboral protegiendo los derechos sociales de los trabajadores y garantizar los derechos de propiedad privada. En esencia, propone fortalecer la LE para lograr la prosperidad nacional.

Sánchez (2011) abordó la importancia que tienen las instituciones formales e informales para el crecimiento económico, señaló que tienen un efecto directo sobre la productividad y uno indirecto sobre la inversión, al mejorar la calidad de las políticas gubernamentales, su transparencia, el Estado de derecho y la contención de la corrupción. En su estudio se comparó la tasa de crecimiento en México con un índice de calidad institucional para el periodo 2007-2010, reportó que la economía se contrajo entre 2008 y 2009 y lo mismo ocurrió con el índice de calidad institucional, mientras que del 2009 al 2010 el crecimiento mejoró, pero el índice retrocedió.

Sánchez (2011) concluye que, para crecer y generar empleos, es necesario que la economía tenga su base en el Estado, democracia, desregulación de los mercados y LE. De manera particular sugiere fomentar la innovación, desregular los mercados de trabajo, mejorar la educación universitaria y reformar el sistema educativo básico, desarrollar el sector salud, crear infraestructura pública, mantener un entorno macroeconómico saludable y ampliar el tamaño de mercado (poder de compra).

Ramírez y Sánchez (2013) realizaron estimaciones en México a nivel de entidad federativa para conocer la correlación existente entre la corrupción y el crecimiento económico al considerar el papel que juegan las instituciones. Usaron información del 2001 al 2010 agrupada en panel, el resultado principal fue que la corrupción afecta positivamente el crecimiento económico. Como parte de sus estimaciones, la única variable que siempre resultó significativa fue el grado de escolaridad con signo positivo. En cuanto a la descripción de los datos destaca la existencia de un bajo crecimiento, un nivel alto de corrupción y medio de calidad institucional.

El grado de criminalidad, al estar relacionado con el Estado de derecho y la garantía de la propiedad privada, es parte de la LE; González (2014), con datos para las treinta y dos entidades federativas de México del 2003 al 2010, estudió la relación entre esto y el crecimiento económico mediante un panel de efectos fijos. Encontró que entre las variables existió una relación inversa, pues, en la medida en que se incrementan los homicidios, robos y lesiones dolosas, el PIB per cápita se reduce.

Isaac Sánchez Juárez y Rosa M. García-Almada

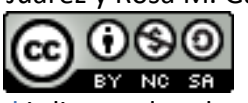

Revista Economía y Sociedad by Universidad Nacional is licensed under a CreativeCommons Reconocimiento-NoComercial- 
Sánchez y García (2014) usaron datos por entidad federativa para el periodo 2001-2008, con el fin de probar el efecto que tres tipos de instituciones formales (derechos de propiedad, calidad gubernamental y tipo de sistema político) tuvieron en el crecimiento económico de México. Encontraron que, a nivel nacional, una deficiente arquitectura institucional condujo a un bajo crecimiento. No obstante, al inspeccionar la relación entre cada una de las variables institucionales y los cambios del PIB per cápita por Estado, no pudieron sostener que las mejoras institucionales se correspondieron con un mayor crecimiento económico.

Finalmente, Chávez, Fonseca y Gómez-Zaldívar (2017) analizaron la relación existente entre el Estado de derecho y el crecimiento económico en las entidades federativas mexicanas del 2006 al 2013. Se centraron en la eficiencia del sistema de justicia y para evaluarlo usaron el tiempo que toma resolver disputas comerciales. Encontraron que una disminución de 100 días en el tiempo promedio que toma resolver disputas comerciales incrementaría 0,6 \% el PIB per cápita en las entidades federativas.

Las escasas evidencias encontradas indican que la LE o alguno de sus componentes relacionados (Estado de derecho, ausencia de corrupción, calidad institucional), en cierta medida, promueven el crecimiento de la producción tal y como se asume en el marco de referencia. Ahora bien, en este artículo, con los datos y los métodos que se presentan enseguida, se indaga cuál es el impacto o tipo de correlación existente entre la LE y el empleo con el objetivo de contribuir a la toma de decisiones por parte de los principales actores públicos, así como ampliar el conocimiento científico existente.

\section{Fuente de los datos y métodos}

En el estudio se contó con una variable dependiente que fue el empleo formal y cuatro variables independientes: índice de LE, producción no manufacturera, producción manufacturera y una variable dicotómica (crisis). Todas las variables, para el periodo 2003-2015, se analizaron en treinta y dos entidades federativas. ${ }^{5}$ La variable de empleo formal (LA) se obtuvo del Banco de Información Económica del INEGI y son datos que toma del Instituto Mexicano del Seguro social, considera tanto a los grupos trabajadores permanentes como eventuales.

El índice de LE por entidad federativa fue construido por Stansel, Torra y McMahon (2017), investigadores asociados al Fraser Institute, un centro de investigación privado en los Estados Unidos de Norteamérica. Dicho índice para el caso mexicano se compone de tres subcomponentes: 1) Gasto gubernamental (incluye gasto en consumo como porcentaje del ingreso personal; transferencias y subsidios como porcentaje del ingreso personal y pago de pensiones y seguro como porcentaje del ingreso personal). 2) Impuestos (incluye impuestos sobre

${ }^{5}$ En el apéndice 1 se proporcionan los datos brutos usados en las estimaciones.

Isaac Sánchez Juárez y Rosa M. García-Almada

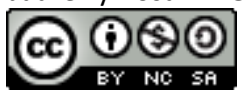

Revista Economía y Sociedad by Universidad Nacional is licensed under a CreativeCommons Reconocimiento-NoComercial- 
ingresos y nómina como porcentaje del ingreso personal; tasa máxima de impuesto sobre la renta; umbral superior del impuesto sobre la renta; impuestos sobre la propiedad como porcentaje del ingreso personal e impuestos sobre las ventas como porcentaje del ingreso personal). 3) Libertad del mercado de trabajo (incluye salario mínimo como porcentaje del ingreso per cápita personal; empleos gubernamentales como porcentaje del total de empleos y densidad sindical como porcentaje del total de empleados).

El índice de LE, por su construcción, toma valores entre 0 y 10 , donde el primer valor indica ausencia total de LE, mientras que el segundo indica un grado absoluto de LE. Para construir este índice Stansel, Torra y McMahon (2017) usaron los tres componentes arriba indicados, todos ellos ponderados con el mismo valor (33,3\%).

Para evaluar el impacto del crecimiento económico sobre el empleo, se trabajó con la producción no manufacturera (PIBNMAN) y manufacturera (PIBMAN) como variables explicativas. Dichas variables se obtuvieron del Banco de Información Económica del INEGI en millones de pesos a precios de 2013. La última variable dependiente fue CRISIS, la cual es dicotómica y toma el valor de uno en los años 2008, 2009 y cero en caso contrario.

Además de contrastar la hipótesis con datos para las treinta y dos entidades federativas, se decidió crear, con fines comparativos, dos regiones que históricamente han sido económicamente contrastantes (la regionalización se tomó de Almonte, Morales y Carbajal, 2018): Frontera Norte (Baja California, Sonora, Chihuahua, Coahuila, Nuevo León y Tamaulipas) y Sur (Campeche, Chiapas, Guerrero, Oaxaca, Quintana Roo, Tabasco, Veracruz y Yucatán). De esta manera se contó con tres paneles, uno con treinta y dos entidades (secciones cruzadas) y trece series de tiempo por tanto 416 observaciones, un segundo para la frontera norte con 78 observaciones y un tercero para la región sur con 104 observaciones. Las variables se trabajaron en logaritmos para poder interpretar los resultados como elasticidades. El modelo general considerado se expresa de la siguiente forma:

$$
\begin{gathered}
\operatorname{LOGLA}_{i t}=f\left\{\text { LOGLE }_{i t} ; \text { LOGPIBNMAN }_{i t} ; \text { LOGPIBMAN }_{i t} ; \text { CRISIS }_{i t j}\right\} \\
\forall \quad \begin{array}{c}
i=1,2, \ldots, 32 ; \\
t=2003, \ldots, 2015
\end{array}
\end{gathered}
$$

CRISIS es una variable dicotómica $i$, con la serie de tiempo $t$, que toma los valores $j$ siguientes:

$$
j=1 \underset{t}{\Leftrightarrow} 2008 \text { y } 2009 \quad t<2008 \therefore j=0 \therefore t>2009
$$

De manera empírica el modelo se representa como:

$$
L_{O G L A}=\beta_{0}+\beta_{1} L O G L E_{i t}+\beta_{2} L O G P I B N M A N_{i t}+\beta_{3} L O G P I B M A N_{i t}+\beta_{4} \text { CRISIS }_{i t j}+\varepsilon_{i t}
$$

Isaac Sánchez Juárez y Rosa M. García-Almada

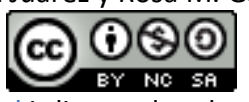

Revista Economía y Sociedad by Universidad Nacional is licensed under a CreativeCommons Reconocimiento-NoComercial- 
Se debe especificar que $B_{0}$ es la constante de la ecuación, así como $\varepsilon_{i t}$ representa los errores aleatorios recogidos por el modelo; para el resto de los coeficientes empíricamente el modelo intenta poner a prueba lo siguiente:

$$
\begin{gathered}
e\left(\text { LOGLA }_{i t} / \text { LOGLE }_{i t}\right)>0: \beta_{1} \\
e\left(\text { LOGLA }_{i t} / \text { LOGPIBNMAN }_{i t}\right)>0: \beta_{2} \\
e\left(\text { LOGLA }_{i t} / \text { LOGPIBMAN }_{i t}\right)>0: \beta_{3} \\
e\left(\text { LOGLA }_{i t} / \text { CRISIS }_{i t j}\right)<0: \beta_{4}
\end{gathered}
$$

De acuerdo con el marco de referencia, se espera un signo positivo para el coeficiente $\boldsymbol{B}_{1}$. Por otro lado, se espera que los incrementos en la producción no manufacturera estén asociados a una mayor generación de puestos de trabajo, un mayor dinamismo de la economía se refleje en empleo formal, por ello el signo esperado de $b_{2}$ es positivo. ${ }^{6}$

Se incluyó, como factor determinante del empleo formal, la producción manufacturera, ya que, desde un punto de vista estructural, en específico kaldoriano, las industrias son el motor de la economía, debido a que dichas actividades incorporan progreso tecnológico y rendimientos crecientes. El signo esperado de $B_{3}$ es positivo. $^{7}$

Para evaluar el rol que tuvo la crisis financiera internacional sobre las economías estatales y regionales en México, se usó la variable ficticia CRISIS que asume el valor de 1 para los años 2008 y 2009 , por lo tanto, se espera que $B_{4}$ sea negativo.

Aclarado el origen de los datos y sus transformaciones, así como los signos esperados, se procedió a realizar las estimaciones para lo cual, en el caso de las treinta y dos entidades federativas, se usó, en primera instancia, la estimación mediante un panel de efectos fijos, enseguida un panel con efectos aleatorios (ambos en la sección cruzada). A partir de las dos estimaciones se aplicó la prueba de Hausman para determinar qué estimación se tomaría en cuenta. ${ }^{8}$

Dado que las estimaciones de MCO con efectos fijos o aleatorios tienen algunos problemas de consistencia y posible sesgo, aprovechando que se cuenta con un periodo corto de tiempo, pero un relativamente amplio número de secciones cruzadas, se procedió a aplicar el método generalizado de momentos (GMM) propuesto por Arellano y Bond (1991), el cual permite tener estimaciones en panel robustas. La validez de los instrumentos en esta clase de estimaciones se

\footnotetext{
${ }^{6}$ Sobre la correlación positiva entre la producción y el empleo revisar Bracamontes y Camberos (2016).

${ }^{7}$ Respecto a esto ver Calderón y Sánchez (2012); Sánchez y Moreno-Brid (ㅁ16) y Carbajal, Carrillo y Almonte (2018).

${ }^{8}$ Para mayores referencias sobre estos métodos ver Almonte, Morales y Carbajal (2018, pp. 203-205).
} 
verifica por medio del estadístico $J$ y su valor de probabilidad, si esta es elevada, entonces el modelo estimado resulta adecuado. ${ }^{9}$ En el caso de las regiones frontera norte y sur, únicamente se realizaron estimaciones mediante $\mathrm{MCO}$ con efectos fijos y aleatorios. Por lo que en este artículo se analiza el total de entidades y dos regiones que resultan de interés por su notorio contraste económico-histórico.

\section{Descripción de los datos y resultados econométricos}

Para facilitar la interpretación de los datos, se agrupó en cuartiles la información relativa a la tasa media anual de crecimiento del empleo formal (ver Figura 2). Destaca que no se registró decrecimiento en el periodo para ningún Estado. Entre los Estados con crecimiento muy bajo (Q1) interesan: Chihuahua (frontera norte), Guerrero (sur), Tamaulipas (frontera norte), Veracruz (sur) y Yucatán (sur). En el otro extremo se encuentran los Estados con crecimiento elevado (Q4): Quintana Roo (sur) y Tabasco (sur). Con un crecimiento bajo (Q2) se tuvo a los estados de Baja California (frontera norte), Coahuila (frontera norte) y Oaxaca (sur); mientras que con un crecimiento moderado (Q3) a Nuevo León (frontera norte), Sonora (frontera norte), Campeche (sur) y Chiapas (sur).

En promedio, la tasa de crecimiento del empleo formal para todo el país fue de 3,18\%, mientras que para la región frontera norte fue $2,78 \%$ y para la sur 3,18 \% mismo valor que el nacional. En el caso de la frontera norte, la entidad que más empleos formales registró en 2015 fue Nuevo León con 1414 693, mientras que en la Sur Veracruz con 733 299. En cuanto a la tasa de crecimiento 2003-2015, el mayor incremento en la frontera norte se observó en Sonora con 3,59 $\%$ y en la sur en Quintana Roo 5,02 \%, muy cerca del valor máximo registrado por el Estado de Querétaro con 5,17\%. La entidad con el menor crecimiento en el país fue Tlaxcala, con un valor de 1,28\%. Por otro lado, la Ciudad de México fue la entidad que más empleos formales reportó 3112585 y tuvo un crecimiento de 3,01\%.

En cuanto a la LE a nivel nacional, en 2003 se ubicó en 6,6, mientras que, en 2015 en 6,2, su valor máximo se presentó en 2004 con 6,7 para luego reducirse paulatinamente. Observe la correspondencia entre la baja tasa de generación de empleo formal y el decrecimiento de la LE. Ahora bien, al igual que se realizó con el empleo, con los niveles del índice de LE se crearon cuartiles para el año 2015 (ver Figura 3). En el Q1 de LE muy baja se ubicaron los Estados de: Chihuahua (frontera norte), Campeche (sur) y Guerrero (sur). En Q2 de LE baja se ubicaron Nuevo León (frontera norte), Chiapas (sur), Oaxaca (sur) y Quintana Roo (sur). En Q3 de LE moderada Tamaulipas (frontera norte), Tabasco (sur), Veracruz (sur) y Yucatán (sur). Clasificados en Q4 de mayor LE se ubicaron Baja California, Coahuila y Sonora (Frontera Norte).

\footnotetext{
${ }^{9}$ Para referencias sobre el GMM ver Judson y Owen (1999).
}

Isaac Sánchez Juárez y Rosa M. García-Almada

Revista Economía y Sociedad by Universidad Nacional is licensed under a CreativeCommons Reconocimiento-NoComercial- 


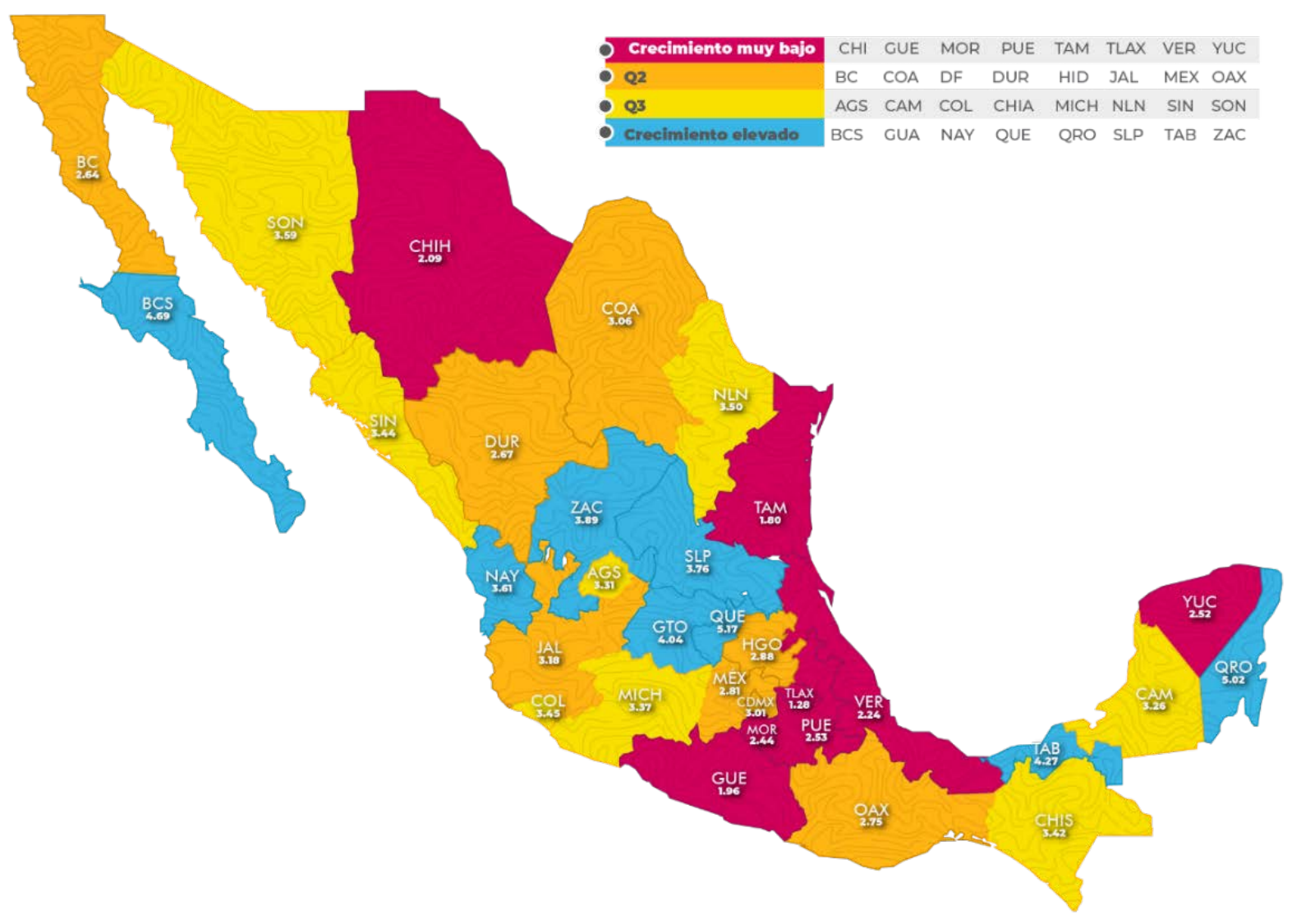

Figura 2. Agrupación de las entidades, TMAC del empleo formal, 2003-2015, México. Fuente: Elaboración propia a partir de los datos del INEGI.

Como se observa en la Figura 3, ningún Estado de la región sur fue considerado como muy libre, cinco de ocho Estados se clasificaron como poco libres. En 2015 el Estado con mayor LE fue Jalisco con un índice de 6,5, mientras que el menos libre fue la Ciudad de México con 5-5. Tanto la Ciudad de México como Jalisco observaron un crecimiento bajo del empleo formal. En el caso de la región sur, como se indicó antes, Quintana Roo fue donde más creció el empleo, a pesar de tener un nivel bajo de LE. Por otro lado, en la frontera norte, Sonora que fue el Estado donde más creció el empleo, también presentó un índice elevado de LE, mientras que Chihuahua con una baja LE relativa registró una muy baja tasa de crecimiento del empleo formal. 


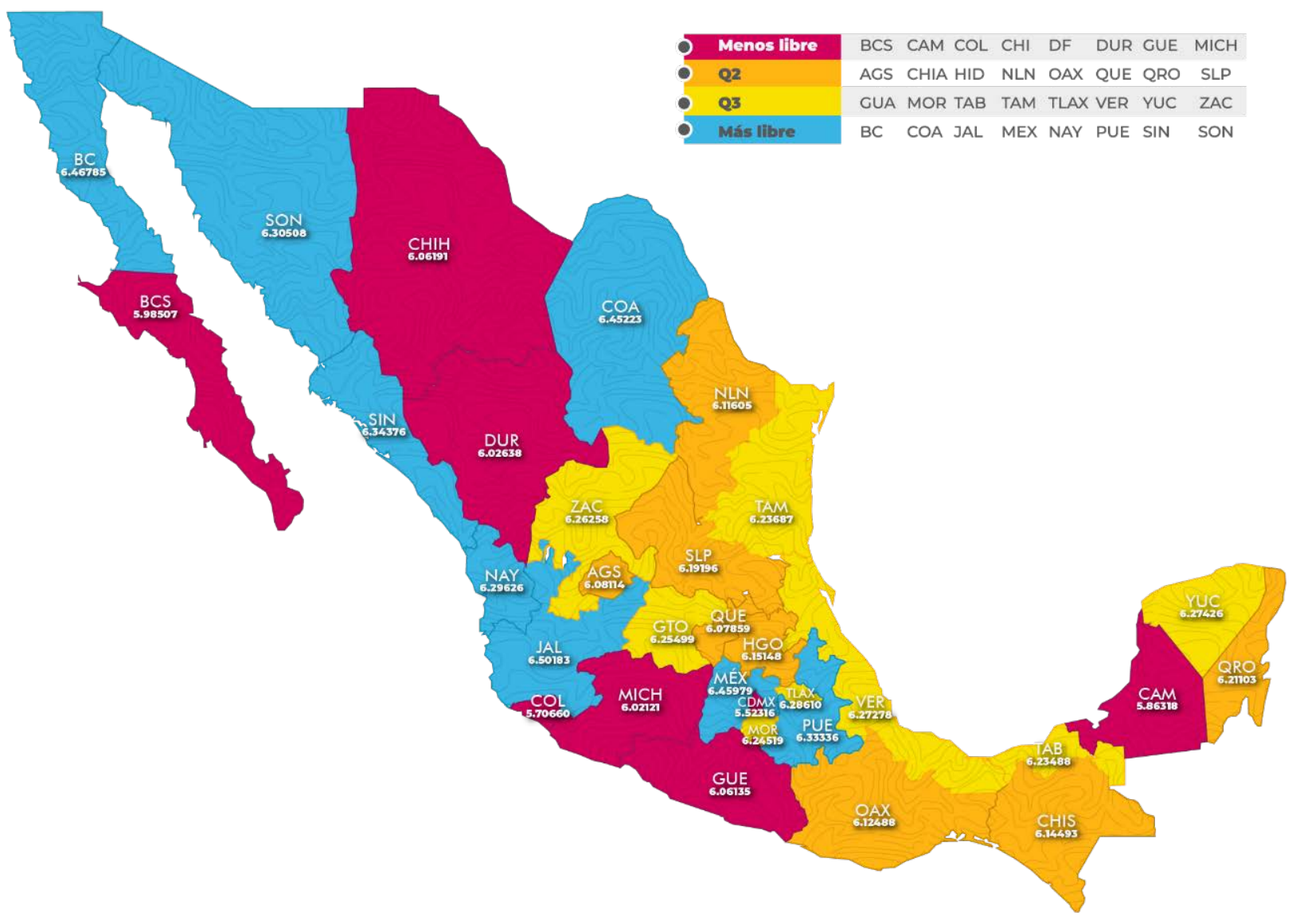

Figura 3. Agrupación de las entidades, nivel de LE 2015, México. Fuente: Elaboración propia con base en datos de Stansel, Torra y McMahon (2017).

En la Tabla 1 se presenta la matriz de correlaciones entre las variables involucradas. Se observa que entre el LOGLA y LOGLE existe una correlación positiva, pero no estadísticamente significativa, aunado a que el coeficiente es sumamente reducido; dicho número resume bien la información contenida en las series y la tendencia que se observa entre ellas. Destaca que LOGPIBNMAN y LOGPIBMAN presentan correlaciones positivas con LOGLA que son estadísticamente significativas. La variable CRISIS tiene el signo esperado de la correlación con LOGLA, pero no es significativo.

Otro dato interesante de la Tabla 1 tiene que ver con la correlación negativa entre LOGLE y LOGPIBNMAN, coeficiente estadísticamente significativo. De forma contrastante, la correlación entre LOGLE y LOGPIBMAN es positiva y significativa estadísticamente. En lo que se refiere a CRISIS, se presentó el signo negativo esperado respecto a LOGPIBNMAN y LOGPIBMAN, pero en todos los casos no fue significativo. 
Tabla 1

Matriz de correlaciones entre las variables, 2003-2015, 416 observaciones

\begin{tabular}{lrrrrr}
\hline & LOGLA & \multicolumn{1}{c}{ LOGLE } & LOGPIBNMAN & LOGPIBMAN & CRISIS \\
\hline LOGLA & 1.0000 & & & & \\
LOGLE & 0.0053 & 1.0000 & & & \\
LOGPIBNMAN & $0.8155^{*}$ & $-0.1315^{*}$ & 1.0000 & & \\
LOGPIBMAN & $0.8117^{*}$ & $0.1965^{*}$ & $0.5975^{*}$ & 1.0000 & \\
CRISIS & -0.0116 & $-0.1635^{*}$ & -0.0089 & -0.0113 & 1.0000 \\
\hline
\end{tabular}

Notas: * Coeficientes significativos al 99 \%. Fuente: Elaboración propia.

Teniendo en cuenta la descripción de los datos, se procedió a la realización de las estimaciones econométricas. Lo primero que se hizo fue aplicar la prueba de Hausman para discriminar entre el método de estimación con efectos aleatorios y fijos. Se encontró que el mejor método de estimación era el de efectos aleatorios, los resultados se presentan en la Tabla 2 (la prueba realizada de Hausman se consigna el Apéndice 2).

Tabla 2

Estimaciones con efectos aleatorios (EA, 416 obs.), fijos (EF, 416 obs.) y GMM (352 obs.). 32 entidades, 2003-2015

\section{Variable dependiente LOGLA}

\begin{tabular}{lrrr}
\hline & \multicolumn{1}{c}{$\mathrm{EA}^{+}$} & \multicolumn{1}{c}{$\mathrm{EF}^{+}$} & \multicolumn{1}{c}{$\mathrm{GMM}$} \\
\hline LOGLE & $-0.837143^{* * *}$ & $-0.849098^{* * *}$ & $-0.040414^{* *}$ \\
& $(-8.382821)$ & $(-8.245342)$ & $(-2.331908)$ \\
LOGPIBNMAN & $0.626479^{* * *}$ & $0.635631^{* * *}$ & $0.412919^{* * *}$ \\
& $(18.34199)$ & $(17.66409)$ & $(22.46720)$ \\
LOGPIBMAN & $0.243199^{* * *}$ & $0.216274^{* * *}$ & $0.178979^{* * *}$ \\
& $(8.516096)$ & $(6.487519)$ & $(7.294698)$ \\
CRISIS & $-0.023767^{* * *}$ & $-0.025020^{* * *}$ & $-0.015624^{* * *}$ \\
& $(-2.764130)$ & $(-2.880702)$ & $(-9.675699)$ \\
LOGLA(-1) & - & - & $0.577923^{* * *}$ \\
R $^{2}$ ajustado & & & $(24.28621)$ \\
Estadístico F & 0.780113 & 0.995187 & - \\
Prob. (Estadístico-F) & 369.0834 & 2452.682 & - \\
Estadístico J & 0.000000 & 0.000000 & - \\
Prob. (Estadístico-J) & - & - & 24.37047 \\
\hline
\end{tabular}

Nota: Estadístico t entre paréntesis.

+ Efectos en la sección cruzada.

*** Significativo al $99 \%, * *$ Significativo al $95 \%$.

Fuente: Elaboración propia usando Eviews 7.2. 
El modelo con efectos aleatorios presentó una buena bondad de ajuste, que permite descartar la hipótesis que se desprende del marco de referencia, ya que el coeficiente de LOGLE es negativo, la elasticidad fue -0.83 y resulta estadísticamente significativa (observe que esto también puede decirse usando los resultados con efectos fijos). De igual manera LOGPIBNMAN presenta el signo positivo esperado y es significativo, lo mismo que la producción industrial manufacturera, de esta forma se confirma que el crecimiento se correlaciona positivamente con las variaciones del empleo formal. La variable CRISIS presentó signo negativo estadísticamente significativo, con lo que se asume tuvo efectos nocivos sobre la generación de empleo formal.

Para confirmar los resultados y superar algunas problemáticas en las estimaciones con efectos fijos y aleatorios, se procedió a la estimación de un panel dinámico con GMM (Tabla 2). Se encontró que la variable de rezago LOGLA fue estadísticamente significativa y que los signos reportados en las estimaciones con efectos aleatorios y fijos se mantienen, pero se alteran los valores de las elasticidades, en especial de LOGLE que es -0.04 . Se reduce también el efecto positivo que tienen los incrementos en la producción no manufacturera y manufacturera, mientras que la relación de LOGLA con respecto a CRISIS no se altera (es negativa). La estimación con GMM es estadísticamente robusta, lo que se sabe por el estadístico $J$ y su valor de probabilidad (un rechazo de la hipótesis nula implicaría que los instrumentos no satisfacen las condiciones de ortogonalidad requeridas). ${ }^{10}$

Tabla 3

Estimaciones con efectos aleatorios (EA, 78 obs.) y fijos (EF, 78 obs.). Frontera norte, 2003-2015

\begin{tabular}{lrr}
\hline \multicolumn{3}{l}{ Variable dependiente LOGLA } \\
\hline \multicolumn{1}{c}{$\mathrm{EA}^{+}$} & \multicolumn{1}{c}{$\mathrm{EF}^{+}$} \\
\hline LOGLE & 0.171447 & 0.177238 \\
& $(1.337741)$ & $(1.376139)$ \\
LOGPIBNMAN & $0.870339^{* * *}$ & $0.873426^{* * *}$ \\
& $(13.83073)$ & $(13.73678)$ \\
LOGPIBMAN & $0.171118^{* * *}$ & $0.171715^{* * *}$ \\
& $(3.545414)$ & $(3.529923)$ \\
CRISIS & -0.009093 & -0.008899 \\
& $(-0.915413)$ & $(-0.895419)$ \\
$\mathrm{R}^{2}$ ajustado & 0.916602 & 0.990743 \\
Estadístico F & 212.5698 & 916.6354 \\
Prob. (Estadístico-F) & 0.000000 & 0.000000 \\
\hline
\end{tabular}

Notas: Estadístico t entre paréntesis.

+ Efectos en la sección cruzada.

*** Significativo al $99 \%$.

Fuente: Elaboración propia usando Eviews 7.2.

${ }^{10}$ Ver Baum, Schaffer y Stillman $(\underline{2003}$, p. 16).

Isaac Sánchez Juárez y Rosa M. García-Almada

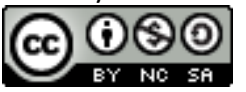

Revista Economía y Sociedad by Universidad Nacional is licensed under a CreativeCommons Reconocimiento-NoComercialCompartirlgual 4.0 Internacional License. Creado a partir de la obra en http://www.revistas.una.ac.cr/index.php/economia 
Como se indicó en la segunda sección, adicional a las estimaciones con la muestra de 32 entidades, se realizaron estimaciones para dos regiones que muestran fuertes contrastes tanto económicos como sociales, las regiones frontera norte y sur. ${ }^{11}$ En el caso de la región frontera norte se estimaron dos modelos: uno con efectos fijos y otro con efectos aleatorios (Tabla 3). Con los dos se concluye que el LOGLE se correlacionó positivamente con LOGLA, con una elasticidad de 0.17 , aunque dicha relación no fue estadísticamente significativa (la prueba de Hausman que permitió elegir el modelo de efectos aleatorios se consigna en el Apéndice 2).

Como en el caso de las treinta y dos entidades, la producción (tanto no manufacturera como manufacturera) se correlacionó positivamente con el empleo formal, en particular un incremento de $100 \%$ en la producción manufacturera provocaba un aumento de $17 \%$ en el empleo formal (ceteris paribus). El coeficiente asociado a la variable CRISIS, aunque presentó el signo esperado, no fue estadísticamente significativo. Los resultados pueden estar asociados a la vinculación que la región tiene, a través de las exportaciones manufactureras, con el resto del mundo particularmente con los Estados Unidos de Norteamérica. ${ }^{12}$

Tabla 4

Estimaciones con efectos aleatorios (EA, 104 obs.) y fijos (EF, 104 obs.). Región sur, 2003-2015

\begin{tabular}{|c|c|c|}
\hline \multicolumn{3}{|c|}{ Variable dependiente LOGLA } \\
\hline & $\mathrm{EA}^{+}$ & $\mathrm{EF}^{+}$ \\
\hline LOGLE & $-2.208606 * * *$ & $-2.185118 * * *$ \\
\hline LOGPIBNMAN & $0.309294 * * *$ & $0.354350 * * *$ \\
\hline LOGPIBMAN & $0.223135^{* * *}$ & 0.171600 \\
\hline CRISIS & $-0.059848 * *$ & $-0.057907 * *$ \\
\hline $\mathrm{R}^{2}$ ajustado & 0.516811 & 0.972472 \\
\hline Estadístico F & 28.54181 & 331.7825 \\
\hline Prob. (Estadístico-F) & 0.000000 & 0.000000 \\
\hline
\end{tabular}

Notas: Estadístico t entre paréntesis.

+ Efectos en la sección cruzada.

*** Significativo al $99 \%, * *$ Significativo al $95 \%$.

Fuente: Elaboración propia usando Eviews 7.2.

En lo que respecta a la región sur, los resultados de las estimaciones se presentan en la Tabla 4 (el modelo elegido de acuerdo con la prueba de Hausman fue el de efectos aleatorios, ver Apéndice 2). Se halló que ante un $100 \%$ de incremento en la LE el empleo formal se reduce 220 $\%$ (ceteris paribus). Al ser una región tradicional con menor contacto con el sector externo y menor participación manufacturera, parece congruente el resultado (es una región que depende

\footnotetext{
${ }^{11}$ Respecto a las diferencias regionales en México y en particular el atraso de la región sur se recomienda la lectura de Dávila, Kessel y Levy (2002).

12 Respecto a la integración de la Frontera Norte con los EE. UU y su impacto en el empleo se recomienda leer a Mendoza (2013).
}

Isaac Sánchez Juárez y Rosa M. García-Almada

Revista Economía y Sociedad by Universidad Nacional is licensed under a CreativeCommons Reconocimiento-NoComercial- 
en mayor medida del gobierno y su intervención). En el caso de la producción, tanto manufacturera como no manufacturera, se observó el signo positivo esperado, por lo que nuevamente se reafirma la importancia de establecer políticas que ayuden al crecimiento de la producción de bienes y servicios. La variable CRISIS presentó el signo negativo esperado y su coeficiente fue estadísticamente significativo; esto implica que la estabilidad macroeconómica se confirma como un elemento a mantener, sujeto al crecimiento económico y a la generación de empleo. ${ }^{13}$

\section{Conclusiones}

En México, a partir de 1982, como resultado de la grave crisis económica (decrecimiento, pérdida del poder adquisitivo, marginación, desempleo), el gobierno federal tomó la decisión de cambiar el modelo económico, se pasó de uno centrado en una fuerte participación estatal en la actividad productiva y una economía cerrada, a otro denominado "neoliberal", caracterizado por la desregulación de la actividad económica, apertura de los mercados al comercio internacional y, en particular, minimización de la influencia del Estado en la economía (en resumen LE). ${ }^{14}$ En teoría esto ayudaría a crecer y generar los empleos formales requeridos por la población.

Los resultados no fueron los esperados, ya que la economía desde entonces no ha logrado crecer más allá del 2,0\% promedio anual si se usa el PIB total y 0,4 \% con el PIB per cápita; la generación de empleo formal también ha estado por debajo de lo requerido y por ello en 2015 había poco más de 50 millones de mexicanos en situación de pobreza. Por otra parte, la LE sí se incrementó (al menos hasta el 2001), sin que eso haya podido corresponderse con un mayor crecimiento económico y empleo formal.

En este artículo, con la información disponible a nivel de entidad federativa, para el periodo 20032015, se presentó evidencia que da cuenta de una correlación negativa entre la LE y el empleo formal. Este hallazgo contrasta con el marco de referencia expuesto en la segunda sección del artículo y es probable que no era lo que tenían en mente los entes tomadores de decisiones que apostaron por este modelo económico. Se advierte que este resultado se encuentra limitado por el periodo de estudio, por los métodos aplicados y por la medida utilizada de LE.

\footnotetext{
${ }^{13}$ Se realizó una estimación más de la ecuación básica presentada en la segunda sección, incluyendo dos variables ficticias para las regiones frontera norte y sur, con datos de las treinta y dos entidades. Se encontró que, en dicha estimación, la relación entre LOGLE y LOGLA era negativa, pero no estadísticamente significativa. Las dos variables ficticias para representar las regiones resultaron significativas y con signos opuestos (frontera norte positivo y sur negativo) lo que confirma que existen diferencias importantes entre estas dos regiones, en particular respecto al impacto que la LE tiene sobre el empleo formal. Dado que no cambian los resultados que hasta aquí se presentan, dicha estimación se envió al Apéndice 3.

${ }^{14}$ Respecto a los mitos del modelo neoliberal se recomienda la lectura de Chang (2009) y para un tratamiento extenso de la historia económica de México desde 1982 al 2015 leer Cárdenas (2015).
}

Isaac Sánchez Juárez y Rosa M. García-Almada

Revista Economía y Sociedad by Universidad Nacional is licensed under a CreativeCommons Reconocimiento-NoComercial- 
La elasticidad del empleo con respecto a la LE en la muestra de treinta y dos entidades fue negativa, con el método de efectos aleatorios -0.83 , con efectos fijos -0.84 y con GMM -0.04 . Conviene recordar que la medida utilizada de LE incorpora tres componentes: 1) gasto público, 2) impuestos y 3) flexibilidad del mercado laboral. El indicador utilizado de LE se acerca a 10 conforme se da una reducción de la intervención del Estado (menos gasto público e impuestos) y se flexibiliza el mercado laboral. Como se reportó la LE en México pasó de 6.6 en 2003 a 6.2 en 2015, tuvo una reducción, que se correspondió con una baja tasa de generación de empleo formal (3,18 \% en promedio del 2003 al 2015). La LE está lejos de 10, su valor máximo. De acuerdo con los resultados econométricos, si aumentara, es probable que esto no ayudaría a crear empleos formales, incluso podría jugar en contra de este objetivo. Por ello se concluye que resulta necesario cambiar el modelo económico, si lo que se pretende es crear más empleo formal. Este estudio aporta evidencia en favor de finalizar el modelo "neoliberal" que se ha aplicado en México.

Los resultados reportados en este artículo contrastan con los publicados por Sánchez (2009) y Sánchez (2011), quien argumentaba que la LE era causa del crecimiento de la producción de bienes y servicios. Por otro lado, confirman lo que encontraron Sánchez y García (2014), quienes sostenían que, con datos estatales, no era posible afirmar que las mejoras en los derechos de propiedad, calidad gubernamental y tipo de sistema político condujeran a un mayor crecimiento.

Con el ánimo de proporcionar mayores evidencias que sustenten la necesidad de cambiar el modelo económico, se estimaron también modelos regionales, en particular para la frontera norte que se caracteriza por su fuerte vinculación con los EE. UU. a través de las exportaciones de manufacturas y la región sur que tiene el mayor número de personas en situación de pobreza, muestra una escasa conexión con la economía americana y se compone de economías enfocadas en el comercio y los servicios. Para la frontera norte, la elasticidad del empleo con respecto a la LE fue positiva (sin ser estadísticamente significativa), por lo que no cambia el resultado general. Por otra parte, para la región sur se reportó, tanto con efectos aleatorios como fijos, una elasticidad negativa de 2.20 y 2.18 respectivamente, lo que refuerza el resultado general.

También, en el artículo se estimó el efecto de la producción de bienes y servicios, tanto del sector manufacturero como del no manufacturero. Los hallazgos confirman que la mejor estrategia para generar empleo formal consiste en crear condiciones para que haya crecimiento en todos los sectores de la economía (los coeficientes asociados a estas variables en todas las estimaciones fueron positivos). Por tanto, es recomendable establecer una política nacional de desarrollo productivo con diferencias sectoriales y regionales. Lo anterior debe ser parte del nuevo modelo económico.

Dicho modelo deberá caracterizarse por una fuerte inversión pública, tanto en obras nuevas como en el mantenimiento de las existentes; una reforma fiscal que permita tener un sistema 
impositivo progresivo y genere los recursos para la atención de las necesidades del sector productivo. El nuevo modelo apostará al desarrollo del sector exportador, pero también buscará fortalecer un núcleo endógeno de empresas nacionales que sean competitivas e innovadoras en el contexto internacional, y que conecte todas las cadenas de producción. Un ingrediente esencial de este nuevo modelo sería el gasto de inversión en ciencia, tecnología e innovación, encaminado a crear fortalezas productivas nacionales. Este nuevo modelo se tendrá que enfocar en impulsar a las micro, pequeñas y medianas empresas con crédito y facilidades fiscales que les permitan crecer y generar empleo bien remunerado.

Específicamente en lo que refiere al mercado laboral, el nuevo modelo económico deberá centrarse en el sector trabajador e instaurar condiciones para que mejore se productividad, salario y bienestar. Frenará la flexiprecarización del mercado de trabajo, lo que aumentará el poder de compra y creará un círculo virtuoso de causación acumulativa. Se recomienda establecer una política de recuperación del poder adquisitivo de la clase trabajadora, basada en mediciones de su productividad y costo de vida. En el nuevo modelo propuesto, el bienestar para los grupos trabajadores se traducirá en bienestar para las empresas. ${ }^{15}$

Por otra parte, al momento de escribir la versión final de este artículo (abril 2020), la economía mexicana atravesaba una emergencia producto de la epidemia del COVID-19, en tan solo 15 días ya se habían perdido 346 mil 878 empleos formales (Presidencia de la República, 2020). Para superar esta etapa se sugiere diseñar programas contracíclicos que sirvan como alivio para las empresas y su personal; en este momento es necesario atenuar los costos económicos de las medidas aplicadas en materia de salud (particularmente la cuarentena y suspensión de actividades económicas-sociales no esenciales).

Este artículo, para la muestra de treinta y dos entidades y para la región sur demostró que una crisis, como la del 2008-2009 tuvo efectos negativos sobre la generación de empleo formal, por lo que son necesarias políticas económicas para evitar que dicho impacto se mantenga en el largo plazo. Con la experiencia del pasado, ante la crisis actual se propone establecer un programa para el cuidado del empleo que transfiera recursos directamente a los sectores trabajadores dependiendo de su nivel de ingreso y que otorgue créditos-facilidades fiscales a las empresas que hacen un esfuerzo para mantener a su personal. También se recomienda reducir, en la medida de lo posible, las contribuciones y pago de servicios públicos por parte de las empresas, particularmente las más pequeñas. Para todo esto se ocupan recursos públicos, por lo que deberá gastarse al menos un año con el compromiso de incrementar la recaudación al finalizar la etapa depresiva.

\footnotetext{
${ }^{15}$ Respecto a las estrategias para el tránsito de un modelo "neoliberal" a un modelo de dinamismo productivo para la generación de empleo formal se recomienda leer Sánchez y García (2019).
} 
Como parte de la agenda de investigación, queda el construir un índice de LE por entidad federativa que abarque un mayor número de ámbitos relacionados con la libertad, como, por ejemplo, la apertura comercial, desregulación, ausencia de corrupción, entre otros. Con una medida más amplia de LE tendría que estimarse nuevamente la correlación entre este indicador y el empleo formal. También es necesario revisar el efecto que la LE tiene sobre la inversión y la productividad total de los factores para comprobar su impacto sobre el crecimiento económico y el empleo, todo esto depende de la disponibilidad de información. Adicional a esto, es necesario evaluar la relación aquí estudiada para todas las regiones del país, ya que, de mantenerse el resultado general presentado, se reforzaría el llamado al establecimiento de un nuevo modelo económico. ${ }^{16}$

Finalmente, este trabajo contribuye con nuevo conocimiento respecto a la relación entre la LE y el empleo formal, pues permite descartar discusiones de política económica que soslayan la importancia de la LE o bien exageran su valor. Con los hallazgos se puede aseverar que en México se requiere cambiar el modelo económico para alentar el desarrollo productivo y el empleo formal. Dicho modelo debe construirse e implementarse evitando dogmatismos y entendiendo que las soluciones pasan por una observación cuidadosa de la realidad, atendiendo a necesidades regionales y condicionamientos sectoriales específicos para transitar del atraso a la modernidad.

\section{Referencias}

Almonte, L., Morales, M. y Carbajal, Y. (2018). Inversión extranjera directa y empleo manufacturero. Un análisis regional con datos de panel para México, 2007-2014. Papeles de Población, 24(96), 187-216. doi: https://doi.org/10.22185/24487147.2018.96.19

Arellano, M. y Bond, S. (1991). Some tests of specification for panel data: Monte Carlo evidence and application to employment Equations. The Review of Economic Studies, 58(2), 277297. doi: https://doi.org/10.2307/2297968

Baum, C., Shaffer, M. y Stillman, S. (2003). Instrumental variables and GMM: Estimation and testing. The Stata Journal, 3(1), 1-31. doi: https://doi.org/10.1177/1536867X0300300101

Bracamontes, J. y Camberos, M. (2016). Análisis regional del crecimiento económico y el empleo en el Estado de Sonora. Nóesis. Revista de Ciencias Sociales y Humanidades, 25(50), 91126. doi: https://doi.org/10.20983/noesis.2016.2.4

\footnotetext{
${ }^{16}$ En términos políticos, el actual presidente de México considera que impulsa una cuarta transformación y el fin del modelo "neoliberal". En lo económico-técnico los resultados distan de esto, ya que las políticas aplicadas se caracterizan esencialmente por la austeridad de las finanzas públicas, el fortalecimiento de la apertura comercial y la ausencia de una estrategia integrada de desarrollo de la planta productiva nacional (política industrial activa).
}

Isaac Sánchez Juárez y Rosa M. García-Almada

Revista Economía y Sociedad by Universidad Nacional is licensed under a CreativeCommons Reconocimiento-NoComercial- 
Calderón, C. y Sánchez, I. (2012). Crecimiento económico y política industrial en México. Revista Latinoamericana De Economía, 43(170), 125-154. doi: https://doi.org/10.22201/iiec.20078951e.2012.170.32138

Carbajal, Y., Carrillo, B. y Almonte, L. (2018). Dinámica productiva del sector automotriz y la manufactura en la frontera norte de México: Un análisis con datos de panel, 1980-2014. Frontera Norte, 30(59), 29-56. doi: http://dx.doi.org/10.17428/rfn.v30i59.511

Cárdenas, E. (2015). El largo curso de la economía mexicana. De 1780 a nuestros días. (Fondo de Cultura Económica. https://elfondoenlinea.com/Detalle.aspx?ctit=003717R

Chang, H. (2009). Bad samaritans: The myth of free trade and the secret history of capitalism. (Edición) Londres: Bloomsbury Publishing PLC. https://www.bloomsbury.com/us/badsamaritans-9781596917385/

Chávez, J., Fonseca, F. y Gómez-Zaldívar, M. (2017). Resoluciones de disputas comerciales y desempeño económico regional en México. Ensayos Revista de Economía, 36(1), 79-93. doi: http://doi.org/10.29105/ensayos36.1-4

Dávila, E., Kessel, G. y Levy, S. (2002). El sur también existe: Un ensayo sobre el desarrollo regional de México. Economía Mexicana, 11(2), 205-260. http://www.economiamexicana.cide.edu/vXIn2.htm

González, S. (2014). Criminalidad y crecimiento económico regional en México. Frontera Norte, 26(51), 75-111. doi: http://dx.doi.org/10.17428/rfn.v26i51.557

Gwartney, J. y Lawson, R. (2003). The concept and measurement of economic freedom. European Journal of Political Economy, 19(3), 405-430. doi: https://doi.org/10.1016/S01762680(03)00007-7

Judson, R. y Owen, A. (1999). Estimating dynamic panel data models: A guide for macroeconomists. Economics Letters, 65(1), 9-15. doi: https://doi.org/10.1016/S01651765(99)00130-5

Mendoza, J. (2013). US-Mexican economic integration and its effects on unemployment in Mexico's Northern Border States. Journal of Borderland Studies, 29(1), 93-108. doi: https://doi.org/10.1080/08865655.2012.751732 
Presidencia de la República (2020). Versión estenográfica de la conferencia de prensa matutina. Miércoles 8 de abril de 2020. Recuperado de https://www.gob.mx/presidencia/es/articulos/version-estenografica-de-la-conferencia-deprensa-matutina-martes-7-de-abril-de-2020-239914?idiom=es

Ramírez, L. y Sánchez, I. (2013). Crecimiento económico, corrupción e instituciones en México. Nóesis. Revista de Ciencias Sociales y Humanidades, 22(43-1), 104-133. doi: https://doi.org/10.20983/noesis.2013.1.4

Sánchez, I. (2009). Libertad económica y crecimiento económico: Teoría y evidencias. En S. Sarmiento (coord.), Cuarto concurso de ensayo caminos de la libertad (30-69). Fundación Caminos de la Libertad y Fomento Cultural Grupo Salinas. Recuperado de: https://docplayer.es/55770094-Libertad-economica-y-crecimiento-economico-teoria-yevidencias.html

Sánchez, I. (2011). Calidad institucional, desaceleración del crecimiento y subdesarrollo regional en México. En L. Gutiérrez y M. Limas (coords.), Nuevos enfoques del desarrollo. Una mirada desde las regiones (47-100). México: Universidad Autónoma de Ciudad Juárez. Recuperado de: $\underline{\text { https://dialnet.unirioja.es/servlet/libro?codigo=555798 }}$

Sánchez, I. y García, R. (2014). México: Crecimiento económico regional y marco institucional. En M. Limas y A. Rodríguez (coords.), Nuevas rutas hacia el bienestar social, económico y medioambiental (55-79). Montevideo: Red Iberoamericana de Estudios del Desarrollo. Recuperado de: https://dialnet.unirioja.es/servlet/libro?codigo=562323

Sánchez I. y Moreno-Brid, J. (2016). El reto del crecimiento económico en México: Industrias manufactureras y política industrial. Revista Finanzas y Política Económica, 8(2), 271-299. doi: https://doi.org/10.14718/revfinanzpolitecon.2016.8.2.4

Sánchez, I. y García, R. (2019). Del estancamiento estabilizador al dinamismo productivo en México. Paradigma Económico, 11(2), 181-203. doi: https://doi.org/10.36677/paradigmaeconomico.v11i2.12509

Stansel, D., Torra, J. y McMahon, F. (2017). Economic Freedom of North America 2017 [Base de datos]. Recuperado de: https://www.fraserinstitute.org/studies/economic-freedom-ofnorth-america-2017 
Apéndice 1. Datos utilizados en el artículo

\begin{tabular}{|c|c|c|c|c|c|}
\hline Entidad & Año & $\begin{array}{l}\text { PIBMAN (millones } \\
\text { de pesos a precios } \\
\text { 2013) }\end{array}$ & LE & LA & $\begin{array}{l}\text { PIBNMAN (millones de } \\
\text { pesos a precios 2013) }\end{array}$ \\
\hline Aguascalientes & 2003 & 24,840 & 6.57956 & 180,214 & 96,358 \\
\hline Aguascalientes & 2004 & 26,356 & 6.68432 & 180,747 & 100,198 \\
\hline Aguascalientes & 2005 & 27,809 & 6.54977 & 187,978 & 101,819 \\
\hline Aguascalientes & 2006 & 31,043 & 6.65009 & 197,168 & 107,069 \\
\hline Aguascalientes & 2007 & 36,272 & 6.48803 & 209,062 & 114,033 \\
\hline Aguascalientes & 2008 & 34,793 & 6.42712 & 208,221 & 116,157 \\
\hline Aguascalientes & 2009 & 30,786 & 6.25670 & 198,345 & 112,468 \\
\hline Aguascalientes & 2010 & 37,699 & 6.12894 & 206,272 & 114,506 \\
\hline Aguascalientes & 2011 & 39,283 & 5.98392 & 211,930 & 119,652 \\
\hline Aguascalientes & 2012 & 41,927 & 6.18284 & 223,156 & 125,779 \\
\hline Aguascalientes & 2013 & 44,876 & 6.08149 & 237,967 & 127,944 \\
\hline Aguascalientes & 2014 & 54,782 & 6.20150 & 252,153 & 136,257 \\
\hline Aguascalientes & 2015 & 57,773 & 6.08114 & 266,372 & 140,402 \\
\hline Baja California & 2003 & 105,062 & 6.77438 & 571,361 & 294,453 \\
\hline Baja California & 2004 & 111,492 & 6.88043 & 600,493 & 311,513 \\
\hline Baja California & 2005 & 111,415 & 6.74165 & 623,461 & 321,593 \\
\hline Baja California & 2006 & 121,639 & 6.80421 & 654,268 & 334,380 \\
\hline Baja California & 2007 & 118,429 & 6.63619 & 668,204 & 343,152 \\
\hline Baja California & 2008 & 111,219 & 6.48066 & 659,996 & 346,338 \\
\hline Baja California & 2009 & 91,268 & 6.41932 & 606,748 & 316,478 \\
\hline Baja California & 2010 & 97,120 & 6.41908 & 625,886 & 331,043 \\
\hline Baja California & 2011 & 94,819 & 6.47630 & 651,463 & 345,882 \\
\hline Baja California & 2012 & 99,629 & 6.52053 & 674,386 & 356,395 \\
\hline Baja California & 2013 & 100,696 & 6.44944 & 693,622 & 364,829 \\
\hline Baja California & 2014 & 108,179 & 6.50354 & 731,679 & 365,183 \\
\hline Baja California & 2015 & 119,688 & 6.46785 & 781,157 & 386,250 \\
\hline Baja California Sur & 2003 & 2,018 & 6.56374 & 82,238 & 74,030 \\
\hline Baja California Sur & 2004 & 1,909 & 6.60688 & 89,243 & 79,637 \\
\hline Baja California Sur & 2005 & 1,976 & 6.54330 & 98,191 & 85,421 \\
\hline Baja California Sur & 2006 & 2,074 & 6.59475 & 110,554 & 91,581 \\
\hline Baja California Sur & 2007 & 2,226 & 6.24861 & 123,611 & 103,973 \\
\hline Baja California Sur & 2008 & 2,199 & 6.10080 & 129,277 & 106,777 \\
\hline Baja California Sur & 2009 & 2,065 & 5.91539 & 117,043 & 106,274 \\
\hline
\end{tabular}




\begin{tabular}{|c|c|c|c|c|c|}
\hline Entidad & Año & $\begin{array}{l}\text { PIBMAN (millones } \\
\text { de pesos a precios } \\
\text { 2013) }\end{array}$ & LE & LA & $\begin{array}{l}\text { PIBNMAN (millones de } \\
\text { pesos a precios 2013) }\end{array}$ \\
\hline Baja California Sur & 2010 & 2,041 & 5.93783 & 115,711 & 108,615 \\
\hline Baja California Sur & 2011 & 2,022 & 6.04566 & 118,771 & 112,685 \\
\hline Baja California Sur & 2012 & 1,988 & 6.10162 & 124,019 & 115,358 \\
\hline Baja California Sur & 2013 & 2,113 & 5.94050 & 130,532 & 112,915 \\
\hline Baja California Sur & 2014 & 2,207 & 6.03204 & 134,719 & 112,664 \\
\hline Baja California Sur & 2015 & 2,203 & 5.98507 & 142,471 & 127,893 \\
\hline Campeche & 2003 & 2,616 & 6.08387 & 98,549 & $1,044,895$ \\
\hline Campeche & 2004 & 2,462 & 6.23866 & 102,444 & $1,057,099$ \\
\hline Campeche & 2005 & 2,425 & 6.09979 & 104,724 & $1,036,109$ \\
\hline Campeche & 2006 & 2,468 & 6.26690 & 110,889 & $1,011,812$ \\
\hline Campeche & 2007 & 2,573 & 6.26512 & 116,358 & 945,003 \\
\hline Campeche & 2008 & 2,734 & 5.97482 & 120,859 & 864,497 \\
\hline Campeche & 2009 & 2,815 & 5.99391 & 124,104 & 777,943 \\
\hline Campeche & 2010 & 2,795 & 5.93580 & 123,791 & 751,174 \\
\hline Campeche & 2011 & 2,859 & 5.92058 & 130,489 & 723,645 \\
\hline Campeche & 2012 & 2,752 & 5.86799 & 144,167 & 712,035 \\
\hline Campeche & 2013 & 2,858 & 5.59529 & 152,435 & 718,227 \\
\hline Campeche & 2014 & 2,860 & 5.82396 & 152,306 & 684,409 \\
\hline Campeche & 2015 & 2,746 & 5.86318 & 144,862 & 635,995 \\
\hline Coahuila & 2003 & 190,336 & 6.65088 & 484,898 & 246,238 \\
\hline Coahuila & 2004 & 198,442 & 6.79774 & 489,995 & 250,702 \\
\hline Coahuila & 2005 & 192,892 & 6.60501 & 498,440 & 265,976 \\
\hline Coahuila & 2006 & 203,715 & 6.66856 & 515,013 & 276,773 \\
\hline Coahuila & 2007 & 206,862 & 6.47023 & 532,447 & 293,617 \\
\hline Coahuila & 2008 & 196,504 & 6.36977 & 537,561 & 301,822 \\
\hline Coahuila & 2009 & 138,716 & 6.25832 & 497,505 & 282,611 \\
\hline Coahuila & 2010 & 189,345 & 6.03637 & 538,604 & 300,607 \\
\hline Coahuila & 2011 & 205,903 & 5.89819 & 583,925 & 317,305 \\
\hline Coahuila & 2012 & 221,832 & 6.02035 & 620,135 & 327,720 \\
\hline Coahuila & 2013 & 214,249 & 6.18629 & 639,063 & 323,958 \\
\hline Coahuila & 2014 & 232,242 & 6.50291 & 662,670 & 333,583 \\
\hline Coahuila & 2015 & 231,750 & 6.45223 & 696,597 & 342,100 \\
\hline Colima & 2003 & 3,181 & 5.86723 & 80,276 & 64,552 \\
\hline Colima & 2004 & 3,422 & 6.03880 & 83,469 & 64,372 \\
\hline Colima & 2005 & 3,420 & 5.97489 & 86,773 & 64,838 \\
\hline
\end{tabular}




\begin{tabular}{|c|c|c|c|c|c|}
\hline Entidad & Año & $\begin{array}{l}\text { PIBMAN (millones } \\
\text { de pesos a precios } \\
\text { 2013) }\end{array}$ & LE & LA & $\begin{array}{l}\text { PIBNMAN (millones de } \\
\text { pesos a precios 2013) }\end{array}$ \\
\hline Colima & 2006 & 3,331 & 6.12394 & 89,843 & 69,202 \\
\hline Colima & 2007 & 3,325 & 5.99567 & 94,566 & 74,201 \\
\hline Colima & 2008 & 3,400 & 5.80759 & 97,227 & 75,554 \\
\hline Colima & 2009 & 3,460 & 5.69464 & 97,430 & 72,987 \\
\hline Colima & 2010 & 3,796 & 5.68179 & 102,878 & 78,196 \\
\hline Colima & 2011 & 3,916 & 5.61211 & 108,948 & 84,029 \\
\hline Colima & 2012 & 3,875 & 5.58859 & 111,702 & 86,665 \\
\hline Colima & 2013 & 3,992 & 5.62517 & 115,462 & 87,430 \\
\hline Colima & 2014 & 3,958 & 5.69374 & 118,054 & 89,750 \\
\hline Colima & 2015 & 4,079 & 5.70660 & 120,610 & 91,279 \\
\hline Chiapas & 2003 & 31,458 & 6.42515 & 145,005 & 216,665 \\
\hline Chiapas & 2004 & 29,418 & 6.48826 & 148,152 & 208,958 \\
\hline Chiapas & 2005 & 27,911 & 6.50538 & 154,886 & 212,368 \\
\hline Chiapas & 2006 & 28,795 & 6.60355 & 165,105 & 219,620 \\
\hline Chiapas & 2007 & 29,438 & 6.35368 & 167,983 & 223,099 \\
\hline Chiapas & 2008 & 28,970 & 6.10305 & 172,900 & 229,320 \\
\hline Chiapas & 2009 & 29,509 & 5.99067 & 181,010 & 227,190 \\
\hline Chiapas & 2010 & 29,816 & 6.03119 & 191,979 & 241,173 \\
\hline Chiapas & 2011 & 30,687 & 6.04854 & 201,008 & 248,760 \\
\hline Chiapas & 2012 & 29,883 & 6.05666 & 210,949 & 254,851 \\
\hline Chiapas & 2013 & 29,023 & 6.02172 & 210,432 & 251,902 \\
\hline Chiapas & 2014 & 30,372 & 6.14100 & 212,304 & 264,786 \\
\hline Chiapas & 2015 & 29,774 & 6.14493 & 217,002 & 260,690 \\
\hline Chihuahua & 2003 & 91,659 & 6.51356 & 616,074 & 268,767 \\
\hline Chihuahua & 2004 & 96,976 & 6.65100 & 624,681 & 279,686 \\
\hline Chihuahua & 2005 & 99,908 & 6.52080 & 649,339 & 289,303 \\
\hline Chihuahua & 2006 & 108,845 & 6.62039 & 681,333 & 310,786 \\
\hline Chihuahua & 2007 & 109,652 & 6.53102 & 697,373 & 324,997 \\
\hline Chihuahua & 2008 & 110,123 & 6.52030 & 676,400 & 330,670 \\
\hline Chihuahua & 2009 & 92,935 & 6.27074 & 603,257 & 308,144 \\
\hline Chihuahua & 2010 & 98,573 & 6.09604 & 636,217 & 319,223 \\
\hline Chihuahua & 2011 & 101,462 & 6.03623 & 653,936 & 325,968 \\
\hline Chihuahua & 2012 & 116,732 & 5.88896 & 684,396 & 342,434 \\
\hline Chihuahua & 2013 & 122,756 & 5.89002 & 715,068 & 353,534 \\
\hline Chihuahua & 2014 & 126,167 & 6.09624 & 744,910 & 360,690 \\
\hline
\end{tabular}

Isaac Sánchez Juárez y Rosa M. García-Almada

(c) (i) (2)

Revista Economía y Sociedad by Universidad Nacional is licensed under a CreativeCommons Reconocimiento-NoComercialCompartirlgual 4.0 Internacional License.

Creado a partir de la obra en http://www.revistas.una.ac.cr/index.php/economia 


\begin{tabular}{|c|c|c|c|c|c|}
\hline Entidad & Año & $\begin{array}{l}\text { PIBMAN (millones } \\
\text { de pesos a precios } \\
\text { 2013) }\end{array}$ & LE & LA & $\begin{array}{l}\text { PIBNMAN (millones de } \\
\text { pesos a precios 2013) }\end{array}$ \\
\hline Chihuahua & 2015 & 135,663 & 6.06191 & 789,338 & 379,524 \\
\hline Ciudad de México & 2003 & 188,796 & 5.88425 & $2,179,960$ & $1,944,134$ \\
\hline Ciudad de México & 2004 & 190,287 & 6.02402 & $2,207,562$ & $2,036,663$ \\
\hline Ciudad de México & 2005 & 191,879 & 5.83897 & $2,277,212$ & $2,066,213$ \\
\hline Ciudad de México & 2006 & 199,453 & 5.91075 & $2,373,295$ & $2,175,270$ \\
\hline Ciudad de México & 2007 & 196,514 & 5.68503 & $2,478,165$ & $2,212,051$ \\
\hline Ciudad de México & 2008 & 193,484 & 5.54247 & $2,529,514$ & $2,256,907$ \\
\hline Ciudad de México & 2009 & 181,706 & 5.43264 & $2,471,500$ & $2,180,810$ \\
\hline Ciudad de México & 2010 & 179,545 & 5.48921 & $2,502,508$ & $2,267,366$ \\
\hline Ciudad de México & 2011 & 174,099 & 5.51542 & $2,615,403$ & $2,359,708$ \\
\hline Ciudad de México & 2012 & 171,185 & 5.54035 & $2,729,188$ & $2,462,750$ \\
\hline Ciudad de México & 2013 & 168,347 & 5.51449 & $2,858,855$ & $2,504,719$ \\
\hline Ciudad de México & 2014 & 165,852 & 5.69289 & $2,978,078$ & $2,564,007$ \\
\hline Ciudad de México & 2015 & 160,931 & 5.52316 & $3,112,585$ & $2,675,609$ \\
\hline Durango & 2003 & 26,359 & 6.59869 & 162,757 & 126,564 \\
\hline Durango & 2004 & 26,587 & 6.73107 & 166,952 & 131,075 \\
\hline Durango & 2005 & 26,787 & 6.57414 & 169,568 & 128,215 \\
\hline Durango & 2006 & 26,197 & 6.53894 & 171,651 & 134,191 \\
\hline Durango & 2007 & 25,644 & 6.38192 & 175,857 & 137,066 \\
\hline Durango & 2008 & 27,721 & 6.15353 & 178,496 & 138,002 \\
\hline Durango & 2009 & 27,232 & 5.94402 & 174,732 & 135,852 \\
\hline Durango & 2010 & 27,706 & 5.92789 & 181,436 & 141,562 \\
\hline Durango & 2011 & 28,926 & 6.09267 & 190,226 & 147,388 \\
\hline Durango & 2012 & 30,979 & 6.04084 & 205,551 & 151,964 \\
\hline Durango & 2013 & 30,842 & 5.99012 & 210,354 & 158,211 \\
\hline Durango & 2014 & 29,579 & 6.08066 & 217,120 & 163,961 \\
\hline Durango & 2015 & 30,369 & 6.02638 & 223,237 & 164,620 \\
\hline Guanajuato & 2003 & 99,078 & 6.75310 & 515,878 & 339,277 \\
\hline Guanajuato & 2004 & 103,818 & 6.86951 & 522,083 & 347,135 \\
\hline Guanajuato & 2005 & 105,891 & 6.76903 & 533,632 & 348,734 \\
\hline Guanajuato & 2006 & 108,224 & 6.84808 & 561,577 & 369,423 \\
\hline Guanajuato & 2007 & 108,033 & 6.67611 & 583,456 & 380,697 \\
\hline Guanajuato & 2008 & 110,694 & 6.47856 & 594,884 & 392,330 \\
\hline Guanajuato & 2009 & 107,990 & 5.99368 & 587,191 & 373,685 \\
\hline Guanajuato & 2010 & 123,391 & 5.95035 & 619,574 & 393,778 \\
\hline
\end{tabular}

Revista Economía y Sociedad by Universidad Nacional is licensed under a CreativeCommons Reconocimiento-NoComercial- 


\begin{tabular}{|c|c|c|c|c|c|}
\hline Entidad & Año & $\begin{array}{l}\text { PIBMAN (millones } \\
\text { de pesos a precios } \\
\text { 2013) }\end{array}$ & LE & LA & $\begin{array}{l}\text { PIBNMAN (millones de } \\
\text { pesos a precios 2013) }\end{array}$ \\
\hline Guanajuato & 2011 & 128,173 & 5.93937 & 652,339 & 419,990 \\
\hline Guanajuato & 2012 & 132,403 & 6.42656 & 687,921 & 438,519 \\
\hline Guanajuato & 2013 & 140,603 & 6.29044 & 725,517 & 453,972 \\
\hline Guanajuato & 2014 & 161,834 & 6.33745 & 778,861 & 459,172 \\
\hline Guanajuato & 2015 & 182,052 & 6.25499 & 829,296 & 479,169 \\
\hline Guerrero & 2003 & 7,012 & 6.36033 & 122,027 & 175,702 \\
\hline Guerrero & 2004 & 7,262 & 6.52818 & 127,097 & 185,296 \\
\hline Guerrero & 2005 & 7,324 & 6.38504 & 131,291 & 187,896 \\
\hline Guerrero & 2006 & 7,003 & 6.49387 & 137,118 & 192,537 \\
\hline Guerrero & 2007 & 6,990 & 6.32014 & 141,925 & 197,890 \\
\hline Guerrero & 2008 & 6,803 & 6.17053 & 145,441 & 201,482 \\
\hline Guerrero & 2009 & 6,406 & 5.80781 & 143,175 & 194,834 \\
\hline Guerrero & 2010 & 6,462 & 5.80437 & 142,967 & 205,429 \\
\hline Guerrero & 2011 & 6,633 & 5.72180 & 142,519 & 207,846 \\
\hline Guerrero & 2012 & 6,582 & 6.12413 & 141,603 & 211,537 \\
\hline Guerrero & 2013 & 6,522 & 6.06289 & 145,925 & 212,290 \\
\hline Guerrero & 2014 & 6,337 & 6.18224 & 152,215 & 222,684 \\
\hline Guerrero & 2015 & 6,234 & 6.06135 & 154,006 & 225,791 \\
\hline Hidalgo & 2003 & 46,145 & 6.46324 & 146,043 & 133,408 \\
\hline Hidalgo & 2004 & 49,492 & 6.61351 & 147,242 & 142,058 \\
\hline Hidalgo & 2005 & 49,818 & 6.40892 & 148,248 & 140,255 \\
\hline Hidalgo & 2006 & 49,392 & 6.45023 & 151,592 & 146,012 \\
\hline Hidalgo & 2007 & 50,903 & 6.21390 & 159,460 & 150,752 \\
\hline Hidalgo & 2008 & 48,609 & 6.03228 & 166,603 & 160,191 \\
\hline Hidalgo & 2009 & 47,844 & 5.97427 & 160,024 & 147,737 \\
\hline Hidalgo & 2010 & 46,896 & 6.01204 & 163,986 & 159,407 \\
\hline Hidalgo & 2011 & 47,377 & 5.94563 & 176,254 & 167,192 \\
\hline Hidalgo & 2012 & 47,864 & 6.01247 & 184,977 & 174,933 \\
\hline Hidalgo & 2013 & 47,490 & 6.00466 & 191,554 & 183,493 \\
\hline Hidalgo & 2014 & 48,900 & 6.21103 & 200,916 & 191,179 \\
\hline Hidalgo & 2015 & 50,062 & 6.15148 & 205,251 & 203,519 \\
\hline Jalisco & 2003 & 186,200 & 6.78426 & $1,034,289$ & 608,758 \\
\hline Jalisco & 2004 & 180,710 & 6.92868 & $1,057,021$ & 638,528 \\
\hline Jalisco & 2005 & 189,985 & 6.76343 & $1,083,163$ & 652,144 \\
\hline Jalisco & 2006 & 204,968 & 6.80549 & $1,132,340$ & 681,042 \\
\hline
\end{tabular}

Isaac Sánchez Juárez y Rosa M. García-Almada

(c) (i) (2)

Revista Economía y Sociedad by Universidad Nacional is licensed under a CreativeCommons Reconocimiento-NoComercialCompartirlgual 4.0 Internacional License.

Creado a partir de la obra en http://www.revistas.una.ac.cr/index.php/economia 


\begin{tabular}{|c|c|c|c|c|c|}
\hline Entidad & Año & $\begin{array}{l}\text { PIBMAN (millones } \\
\text { de pesos a precios } \\
\text { 2013) }\end{array}$ & LE & LA & $\begin{array}{l}\text { PIBNMAN (millones de } \\
\text { pesos a precios 2013) }\end{array}$ \\
\hline Jalisco & 2007 & 204,646 & 6.63837 & $1,186,066$ & 708,494 \\
\hline Jalisco & 2008 & 186,508 & 6.45816 & $1,218,025$ & 732,065 \\
\hline Jalisco & 2009 & 177,015 & 6.40809 & $1,200,709$ & 693,304 \\
\hline Jalisco & 2010 & 191,864 & 6.47320 & $1,242,643$ & 733,508 \\
\hline Jalisco & 2011 & 190,923 & 6.43877 & $1,294,432$ & 762,225 \\
\hline Jalisco & 2012 & 201,183 & 6.41591 & $1,333,225$ & 794,103 \\
\hline Jalisco & 2013 & 211,593 & 6.39144 & $1,382,438$ & 806,986 \\
\hline Jalisco & 2014 & 235,672 & 6.55053 & $1,432,723$ & 826,412 \\
\hline Jalisco & 2015 & 248,522 & 6.50183 & $1,505,361$ & 859,160 \\
\hline México & 2003 & 275,629 & 6.89319 & $1,007,227$ & 772,775 \\
\hline México & 2004 & 274,236 & 7.02544 & $1,013,098$ & 799,605 \\
\hline México & 2005 & 271,491 & 6.85819 & $1,030,412$ & 827,886 \\
\hline México & 2006 & 277,275 & 6.91221 & $1,085,349$ & 873,427 \\
\hline México & 2007 & 274,208 & 6.71813 & $1,147,076$ & 910,450 \\
\hline México & 2008 & 270,913 & 6.49839 & $1,172,957$ & 927,232 \\
\hline México & 2009 & 244,936 & 6.46604 & $1,138,280$ & 893,792 \\
\hline México & 2010 & 266,723 & 6.50934 & $1,186,516$ & 960,090 \\
\hline México & 2011 & 282,736 & 6.51666 & $1,241,545$ & $1,000,712$ \\
\hline México & 2012 & 293,471 & 6.46937 & $1,310,548$ & $1,046,523$ \\
\hline México & 2013 & 289,070 & 6.38353 & $1,330,406$ & $1,076,084$ \\
\hline México & 2014 & 278,377 & 6.31885 & $1,351,056$ & $1,127,137$ \\
\hline México & 2015 & 283,492 & 6.45979 & $1,405,179$ & $1,155,030$ \\
\hline Michoacán & 2003 & 39,771 & 6.80012 & 253,405 & 254,698 \\
\hline Michoacán & 2004 & 42,216 & 6.93112 & 260,402 & 258,806 \\
\hline Michoacán & 2005 & 43,058 & 6.82260 & 268,454 & 262,969 \\
\hline Michoacán & 2006 & 42,993 & 6.95528 & 281,863 & 277,459 \\
\hline Michoacán & 2007 & 45,189 & 6.67397 & 299,476 & 283,083 \\
\hline Michoacán & 2008 & 43,300 & 6.43114 & 311,041 & 291,358 \\
\hline Michoacán & 2009 & 35,014 & 6.30544 & 317,775 & 281,989 \\
\hline Michoacán & 2010 & 39,400 & 6.26808 & 331,813 & 290,367 \\
\hline Michoacán & 2011 & 36,310 & 6.19706 & 343,606 & 306,966 \\
\hline Michoacán & 2012 & 36,948 & 6.18996 & 351,375 & 315,082 \\
\hline Michoacán & 2013 & 39,147 & 6.02104 & 352,200 & 320,319 \\
\hline Michoacán & 2014 & 41,723 & 6.13013 & 361,271 & 341,472 \\
\hline Michoacán & 2015 & 38,894 & 6.02121 & 377,201 & 352,773 \\
\hline
\end{tabular}

Isaac Sánchez Juárez y Rosa M. García-Almada

(c) (1) (1) (2)

Revista Economía y Sociedad by Universidad Nacional is licensed under a CreativeCommons Reconocimiento-NoComercialCompartirlgual 4.0 Internacional License.

Creado a partir de la obra en http://www.revistas.una.ac.cr/index.php/economia 


\begin{tabular}{|c|c|c|c|c|c|}
\hline Entidad & Año & $\begin{array}{l}\text { PIBMAN (millones } \\
\text { de pesos a precios } \\
\text { 2013) }\end{array}$ & LE & LA & $\begin{array}{l}\text { PIBNMAN (millones de } \\
\text { pesos a precios 2013) }\end{array}$ \\
\hline Morelos & 2003 & 27,150 & 6.70588 & 149,488 & 130,906 \\
\hline Morelos & 2004 & 28,301 & 6.84913 & 151,314 & 131,434 \\
\hline Morelos & 2005 & 29,016 & 6.67426 & 154,590 & 142,263 \\
\hline Morelos & 2006 & 28,420 & 6.71372 & 159,857 & 139,758 \\
\hline Morelos & 2007 & 30,254 & 6.56762 & 165,601 & 139,071 \\
\hline Morelos & 2008 & 27,365 & 6.43291 & 167,453 & 142,307 \\
\hline Morelos & 2009 & 25,289 & 6.29304 & 165,940 & 143,060 \\
\hline Morelos & 2010 & 28,514 & 6.29771 & 172,802 & 146,471 \\
\hline Morelos & 2011 & 31,759 & 6.24709 & 180,930 & 142,919 \\
\hline Morelos & 2012 & 33,196 & 6.30585 & 190,374 & 142,522 \\
\hline Morelos & 2013 & 34,218 & 6.04951 & 194,403 & 147,908 \\
\hline Morelos & 2014 & 33,858 & 6.24772 & 196,528 & 150,292 \\
\hline Morelos & 2015 & 35,409 & 6.24519 & 199,534 & 151,064 \\
\hline Nayarit & 2003 & 5,376 & 6.68229 & 83,695 & 70,729 \\
\hline Nayarit & 2004 & 5,322 & 6.77055 & 88,887 & 81,557 \\
\hline Nayarit & 2005 & 5,639 & 6.79197 & 94,006 & 84,631 \\
\hline Nayarit & 2006 & 5,475 & 6.91474 & 100,770 & 86,691 \\
\hline Nayarit & 2007 & 5,515 & 6.56924 & 102,232 & 86,160 \\
\hline Nayarit & 2008 & 5,303 & 6.13479 & 107,187 & 92,990 \\
\hline Nayarit & 2009 & 5,098 & 6.09093 & 105,159 & 87,940 \\
\hline Nayarit & 2010 & 5,463 & 6.05343 & 109,631 & 92,323 \\
\hline Nayarit & 2011 & 5,572 & 6.02310 & 113,155 & 95,133 \\
\hline Nayarit & 2012 & 5,715 & 6.11288 & 117,679 & 95,085 \\
\hline Nayarit & 2013 & 5,743 & 6.14972 & 118,286 & 97,885 \\
\hline Nayarit & 2014 & 5,852 & 6.26160 & 123,160 & 103,416 \\
\hline Nayarit & 2015 & 5,949 & 6.29626 & 128,062 & 108,934 \\
\hline Nuevo León & 2003 & 213,897 & 6.56224 & 936,175 & 589,991 \\
\hline Nuevo León & 2004 & 227,011 & 6.67484 & 951,207 & 622,831 \\
\hline Nuevo León & 2005 & 231,929 & 6.53011 & 985,535 & 653,510 \\
\hline Nuevo León & 2006 & 244,764 & 6.59713 & $1,043,850$ & 702,074 \\
\hline Nuevo León & 2007 & 248,569 & 6.36944 & $1,100,515$ & 756,067 \\
\hline Nuevo León & 2008 & 248,597 & 6.22474 & $1,144,379$ & 771,770 \\
\hline Nuevo León & 2009 & 233,723 & 6.17729 & $1,099,028$ & 719,003 \\
\hline Nuevo León & 2010 & 259,654 & 6.20050 & $1,158,648$ & 765,530 \\
\hline Nuevo León & 2011 & 262,629 & 6.17822 & $1,218,471$ & 807,183 \\
\hline
\end{tabular}

Isaac Sánchez Juárez y Rosa M. García-Almada

(c) (i) (8) (2)

Revista Economía y Sociedad by Universidad Nacional is licensed under a CreativeCommons Reconocimiento-NoComercialCompartirlgual 4.0 Internacional License.

Creado a partir de la obra en http://www.revistas.una.ac.cr/index.php/economia 


\begin{tabular}{|c|c|c|c|c|c|}
\hline Entidad & Año & $\begin{array}{l}\text { PIBMAN (millones } \\
\text { de pesos a precios } \\
\text { 2013) }\end{array}$ & LE & LA & $\begin{array}{l}\text { PIBNMAN (millones de } \\
\text { pesos a precios 2013) }\end{array}$ \\
\hline Nuevo León & 2012 & 271,034 & 6.10334 & $1,266,925$ & 842,784 \\
\hline Nuevo León & 2013 & 267,103 & 5.73804 & $1,298,658$ & 857,897 \\
\hline Nuevo León & 2014 & 273,737 & 6.13748 & $1,347,389$ & 888,328 \\
\hline Nuevo León & 2015 & 284,646 & 6.11605 & $1,414,693$ & 934,641 \\
\hline Oaxaca & 2003 & 29,974 & 6.57416 & 139,845 & 172,990 \\
\hline Oaxaca & 2004 & 29,636 & 6.67145 & 140,834 & 181,121 \\
\hline Oaxaca & 2005 & 30,062 & 6.51001 & 144,924 & 183,616 \\
\hline Oaxaca & 2006 & 30,590 & 6.48383 & 147,966 & 187,433 \\
\hline Oaxaca & 2007 & 30,621 & 6.37819 & 150,275 & 189,194 \\
\hline Oaxaca & 2008 & 30,966 & 6.24400 & 154,547 & 195,668 \\
\hline Oaxaca & 2009 & 30,807 & 6.08392 & 158,781 & 193,704 \\
\hline Oaxaca & 2010 & 27,874 & 6.12964 & 160,814 & 200,216 \\
\hline Oaxaca & 2011 & 29,051 & 6.09069 & 164,346 & 205,905 \\
\hline Oaxaca & 2012 & 27,087 & 6.07970 & 173,913 & 212,593 \\
\hline Oaxaca & 2013 & 29,315 & 6.04960 & 181,505 & 216,201 \\
\hline Oaxaca & 2014 & 28,912 & 6.15685 & 186,687 & 221,643 \\
\hline Oaxaca & 2015 & 28,303 & 6.12488 & 193,736 & 232,204 \\
\hline Puebla & 2003 & 101,734 & 6.68891 & 395,635 & 294,173 \\
\hline Puebla & 2004 & 99,009 & 6.87321 & 394,113 & 306,899 \\
\hline Puebla & 2005 & 111,491 & 6.84414 & 400,984 & 316,688 \\
\hline Puebla & 2006 & 114,353 & 6.96160 & 404,306 & 332,848 \\
\hline Puebla & 2007 & 122,563 & 6.65063 & 413,270 & 343,256 \\
\hline Puebla & 2008 & 121,451 & 6.40032 & 421,230 & 347,519 \\
\hline Puebla & 2009 & 100,899 & 6.33045 & 413,728 & 331,679 \\
\hline Puebla & 2010 & 114,657 & 6.44051 & 434,227 & 355,311 \\
\hline Puebla & 2011 & 121,885 & 6.34658 & 453,228 & 371,468 \\
\hline Puebla & 2012 & 132,742 & 6.35354 & 479,021 & 391,484 \\
\hline Puebla & 2013 & 120,107 & 6.20763 & 494,014 & 399,149 \\
\hline Puebla & 2014 & 119,706 & 6.30916 & 508,133 & 404,601 \\
\hline Puebla & 2015 & 122,131 & 6.33336 & 533,811 & 417,316 \\
\hline Querétaro & 2003 & 62,146 & 6.42530 & 253,674 & 149,960 \\
\hline Querétaro & 2004 & 68,160 & 6.54433 & 264,470 & 159,757 \\
\hline Querétaro & 2005 & 71,624 & 6.48852 & 272,627 & 171,687 \\
\hline Querétaro & 2006 & 71,592 & 6.62745 & 290,659 & 186,857 \\
\hline Querétaro & 2007 & 74,563 & 6.47748 & 306,907 & 197,059 \\
\hline
\end{tabular}




\begin{tabular}{|c|c|c|c|c|c|}
\hline Entidad & Año & $\begin{array}{l}\text { PIBMAN (millones } \\
\text { de pesos a precios } \\
\text { 2013) }\end{array}$ & LE & LA & $\begin{array}{l}\text { PIBNMAN (millones de } \\
\text { pesos a precios 2013) }\end{array}$ \\
\hline Querétaro & 2008 & 71,960 & 6.31156 & 317,993 & 206,388 \\
\hline Querétaro & 2009 & 65,001 & 6.14706 & 308,622 & 205,311 \\
\hline Querétaro & 2010 & 74,195 & 6.11786 & 335,653 & 213,209 \\
\hline Querétaro & 2011 & 80,660 & 6.24158 & 366,501 & 228,205 \\
\hline Querétaro & 2012 & 82,446 & 6.35534 & 395,028 & 235,849 \\
\hline Querétaro & 2013 & 81,285 & 6.11152 & 420,258 & 238,705 \\
\hline Querétaro & 2014 & 90,824 & 6.06694 & 439,139 & 254,829 \\
\hline Querétaro & 2015 & 98,140 & 6.07859 & 464,536 & 271,696 \\
\hline Quintana Roo & 2003 & 3,993 & 6.64297 & 185,304 & 140,240 \\
\hline Quintana Roo & 2004 & 4,212 & 6.67959 & 202,013 & 152,729 \\
\hline Quintana Roo & 2005 & 4,287 & 6.57254 & 223,160 & 159,395 \\
\hline Quintana Roo & 2006 & 4,482 & 6.60796 & 236,996 & 169,882 \\
\hline Quintana Roo & 2007 & 4,509 & 6.36484 & 258,801 & 188,396 \\
\hline Quintana Roo & 2008 & 4,636 & 6.23653 & 271,054 & 198,383 \\
\hline Quintana Roo & 2009 & 4,354 & 6.20417 & 257,052 & 181,317 \\
\hline Quintana Roo & 2010 & 4,213 & 6.24143 & 262,460 & 190,935 \\
\hline Quintana Roo & 2011 & 4,103 & 6.15684 & 270,516 & 201,951 \\
\hline Quintana Roo & 2012 & 3,595 & 6.24578 & 277,369 & 212,115 \\
\hline Quintana Roo & 2013 & 3,495 & 6.07807 & 291,690 & 221,778 \\
\hline Quintana Roo & 2014 & 3,705 & 6.26552 & 309,761 & 229,956 \\
\hline Quintana Roo & 2015 & 3,785 & 6.21103 & 333,598 & 241,727 \\
\hline San Luis Potosí & 2003 & 53,876 & 6.58199 & 239,099 & 170,404 \\
\hline San Luis Potosí & 2004 & 59,357 & 6.66947 & 245,155 & 178,107 \\
\hline San Luis Potosí & 2005 & 60,272 & 6.65495 & 258,007 & 186,968 \\
\hline San Luis Potosí & 2006 & 61,731 & 6.78841 & 272,608 & 196,918 \\
\hline San Luis Potosí & 2007 & 62,624 & 6.59113 & 281,803 & 201,692 \\
\hline San Luis Potosí & 2008 & 61,744 & 6.30992 & 287,267 & 208,280 \\
\hline San Luis Potosí & 2009 & 56,537 & 6.19314 & 279,686 & 199,309 \\
\hline San Luis Potosí & 2010 & 64,426 & 6.14661 & 292,021 & 204,971 \\
\hline San Luis Potosí & 2011 & 70,249 & 6.06653 & 310,548 & 213,633 \\
\hline San Luis Potosí & 2012 & 76,613 & 6.08204 & 327,936 & 220,681 \\
\hline San Luis Potosí & 2013 & 77,158 & 6.04456 & 344,558 & 230,738 \\
\hline San Luis Potosí & 2014 & 81,388 & 6.18649 & 356,708 & 234,007 \\
\hline San Luis Potosí & 2015 & 83,382 & 6.19196 & 372,282 & 246,781 \\
\hline Sinaloa & 2003 & 21,850 & 6.73616 & 313,442 & 246,397 \\
\hline
\end{tabular}




\begin{tabular}{|c|c|c|c|c|c|}
\hline Entidad & Año & $\begin{array}{l}\text { PIBMAN (millones } \\
\text { de pesos a precios } \\
\text { 2013) }\end{array}$ & LE & LA & $\begin{array}{l}\text { PIBNMAN (millones de } \\
\text { pesos a precios 2013) }\end{array}$ \\
\hline Sinaloa & 2004 & 23,024 & 6.87581 & 319,283 & 261,634 \\
\hline Sinaloa & 2005 & 24,026 & 6.68230 & 331,285 & 261,682 \\
\hline Sinaloa & 2006 & 23,294 & 6.70248 & 346,730 & 271,658 \\
\hline Sinaloa & 2007 & 23,760 & 6.58820 & 363,482 & 281,862 \\
\hline Sinaloa & 2008 & 23,769 & 6.45178 & 385,677 & 292,612 \\
\hline Sinaloa & 2009 & 24,170 & 6.17567 & 382,045 & 278,896 \\
\hline Sinaloa & 2010 & 24,471 & 6.14502 & 395,035 & 288,184 \\
\hline Sinaloa & 2011 & 25,346 & 6.16448 & 403,075 & 293,417 \\
\hline Sinaloa & 2012 & 26,605 & 6.14916 & 420,450 & 303,586 \\
\hline Sinaloa & 2013 & 25,687 & 6.15634 & 430,670 & 308,411 \\
\hline Sinaloa & 2014 & 26,954 & 6.32803 & 447,112 & 314,258 \\
\hline Sinaloa & 2015 & 28,755 & 6.34376 & 470,437 & 333,150 \\
\hline Sonora & 2003 & 87,048 & 6.71913 & 345,874 & 278,486 \\
\hline Sonora & 2004 & 91,370 & 6.81812 & 364,365 & 293,114 \\
\hline Sonora & 2005 & 98,841 & 6.66257 & 387,621 & 306,040 \\
\hline Sonora & 2006 & 118,521 & 6.77039 & 415,271 & 311,104 \\
\hline Sonora & 2007 & 111,573 & 6.59647 & 433,017 & 323,824 \\
\hline Sonora & 2008 & 115,135 & 6.38968 & 435,401 & 321,582 \\
\hline Sonora & 2009 & 100,622 & 6.16910 & 413,366 & 309,753 \\
\hline Sonora & 2010 & 106,853 & 6.06018 & 436,599 & 324,649 \\
\hline Sonora & 2011 & 117,796 & 6.09465 & 459,167 & 353,714 \\
\hline Sonora & 2012 & 120,953 & 6.25499 & 487,729 & 374,973 \\
\hline Sonora & 2013 & 129,359 & 6.17825 & 506,427 & 380,956 \\
\hline Sonora & 2014 & 131,804 & 6.32718 & 515,360 & 387,279 \\
\hline Sonora & 2015 & 135,963 & 6.30508 & 527,934 & 401,535 \\
\hline Tabasco & 2003 & 48,384 & 6.30181 & 118,669 & 326,507 \\
\hline Tabasco & 2004 & 51,906 & 6.43430 & 121,054 & 339,337 \\
\hline Tabasco & 2005 & 50,209 & 6.37640 & 127,037 & 370,871 \\
\hline Tabasco & 2006 & 49,852 & 6.51378 & 136,003 & 395,457 \\
\hline Tabasco & 2007 & 46,021 & 6.26421 & 140,934 & 408,058 \\
\hline Tabasco & 2008 & 44,836 & 6.10354 & 149,436 & 430,367 \\
\hline Tabasco & 2009 & 44,391 & 5.92729 & 152,135 & 451,553 \\
\hline Tabasco & 2010 & 47,147 & 5.91550 & 157,492 & 477,865 \\
\hline Tabasco & 2011 & 47,572 & 6.12049 & 166,153 & 502,179 \\
\hline Tabasco & 2012 & 47,050 & 6.16247 & 180,182 & 516,954 \\
\hline
\end{tabular}

Isaac Sánchez Juárez y Rosa M. García-Almada

(c) (i) (2)

Revista Economía y Sociedad by Universidad Nacional is licensed under a CreativeCommons Reconocimiento-NoComercialCompartirlgual 4.0 Internacional License.

Creado a partir de la obra en http://www.revistas.una.ac.cr/index.php/economia 


\begin{tabular}{|c|c|c|c|c|c|}
\hline Entidad & Año & $\begin{array}{l}\text { PIBMAN (millones } \\
\text { de pesos a precios } \\
\text { 2013) }\end{array}$ & LE & LA & $\begin{array}{l}\text { PIBNMAN (millones de } \\
\text { pesos a precios 2013) }\end{array}$ \\
\hline Tabasco & 2013 & 49,048 & 6.09763 & 190,027 & 504,580 \\
\hline Tabasco & 2014 & 46,389 & 6.25566 & 197,133 & 516,437 \\
\hline Tabasco & 2015 & 40,691 & 6.23488 & 195,903 & 518,377 \\
\hline Tamaulipas & 2003 & 89,011 & 6.27646 & 497,375 & 302,563 \\
\hline Tamaulipas & 2004 & 99,791 & 6.31280 & 512,827 & 310,322 \\
\hline Tamaulipas & 2005 & 106,730 & 6.26046 & 539,143 & 329,761 \\
\hline Tamaulipas & 2006 & 110,678 & 6.35534 & 563,962 & 338,931 \\
\hline Tamaulipas & 2007 & 112,915 & 6.08998 & 575,768 & 351,093 \\
\hline Tamaulipas & 2008 & 124,247 & 5.92875 & 580,816 & 359,104 \\
\hline Tamaulipas & 2009 & 109,552 & 5.86322 & 539,869 & 330,187 \\
\hline Tamaulipas & 2010 & 105,094 & 5.86420 & 554,994 & 343,121 \\
\hline Tamaulipas & 2011 & 102,912 & 5.91252 & 558,234 & 353,857 \\
\hline Tamaulipas & 2012 & 105,218 & 6.08308 & 573,366 & 361,154 \\
\hline Tamaulipas & 2013 & 105,978 & 6.01156 & 586,766 & 367,263 \\
\hline Tamaulipas & 2014 & 105,938 & 6.20769 & 604,020 & 372,612 \\
\hline Tamaulipas & 2015 & 108,957 & 6.23687 & 616,184 & 381,656 \\
\hline Tlaxcala & 2003 & 20,735 & 6.99607 & 71,742 & 62,519 \\
\hline Tlaxcala & 2004 & 22,486 & 7.07826 & 71,566 & 67,304 \\
\hline Tlaxcala & 2005 & 18,156 & 6.84108 & 72,967 & 61,124 \\
\hline Tlaxcala & 2006 & 19,567 & 6.67744 & 72,279 & 58,323 \\
\hline Tlaxcala & 2007 & 19,227 & 6.49671 & 71,051 & 59,792 \\
\hline Tlaxcala & 2008 & 18,918 & 6.28078 & 69,776 & 64,328 \\
\hline Tlaxcala & 2009 & 17,736 & 6.20611 & 64,598 & 64,003 \\
\hline Tlaxcala & 2010 & 19,041 & 6.18148 & 68,386 & 69,769 \\
\hline Tlaxcala & 2011 & 20,975 & 6.18909 & 71,319 & 65,057 \\
\hline Tlaxcala & 2012 & 21,298 & 6.24831 & 74,748 & 68,621 \\
\hline Tlaxcala & 2013 & 20,999 & 6.19727 & 78,290 & 66,658 \\
\hline Tlaxcala & 2014 & 21,258 & 6.33438 & 79,439 & 69,238 \\
\hline Tlaxcala & 2015 & 21,738 & 6.28610 & 83,617 & 74,871 \\
\hline Veracruz & 2003 & 117,880 & 6.50707 & 562,432 & 495,710 \\
\hline Veracruz & 2004 & 121,464 & 6.59633 & 562,544 & 522,396 \\
\hline Veracruz & 2005 & 127,863 & 6.52136 & 571,725 & 521,044 \\
\hline Veracruz & 2006 & 130,978 & 6.60870 & 599,236 & 553,579 \\
\hline Veracruz & 2007 & 131,885 & 6.43591 & 622,361 & 573,723 \\
\hline Veracruz & 2008 & 133,238 & 6.28994 & 634,955 & 571,076 \\
\hline
\end{tabular}




\begin{tabular}{|c|c|c|c|c|c|}
\hline Entidad & Año & $\begin{array}{c}\text { PIBMAN (millones } \\
\text { de pesos a precios } \\
\text { 2013) }\end{array}$ & LE & LA & $\begin{array}{l}\text { PIBNMAN (millones de } \\
\text { pesos a precios 2013) }\end{array}$ \\
\hline Veracruz & 2009 & 123,874 & 6.19165 & 646,716 & 565,107 \\
\hline Veracruz & 2010 & 125,970 & 6.17383 & 669,530 & 592,179 \\
\hline Veracruz & 2011 & 129,399 & 6.13372 & 683,906 & 617,419 \\
\hline Veracruz & 2012 & 130,876 & 6.21564 & 719,055 & 648,854 \\
\hline Veracruz & 2013 & 134,730 & 6.14168 & 732,329 & 646,628 \\
\hline Veracruz & 2014 & 138,042 & 6.31990 & 732,916 & 652,815 \\
\hline Veracruz & 2015 & 133,473 & 6.27278 & 733,299 & 670,510 \\
\hline Yucatán & 2003 & 19,791 & 6.64206 & 240,987 & 141,846 \\
\hline Yucatán & 2004 & 21,114 & 6.80047 & 245,841 & 147,899 \\
\hline Yucatán & 2005 & 21,714 & 6.60168 & 251,862 & 155,194 \\
\hline Yucatán & 2006 & 23,446 & 6.65233 & 257,246 & 162,017 \\
\hline Yucatán & 2007 & 23,941 & 6.51305 & 263,982 & 167,276 \\
\hline Yucatán & 2008 & 23,783 & 6.30661 & 267,317 & 169,376 \\
\hline Yucatán & 2009 & 23,888 & 6.13232 & 263,662 & 165,478 \\
\hline Yucatán & 2010 & 24,771 & 6.15578 & 271,682 & 171,379 \\
\hline Yucatán & 2011 & 25,369 & 6.12465 & 279,597 & 177,525 \\
\hline Yucatán & 2012 & 25,370 & 6.10528 & 291,888 & 189,331 \\
\hline Yucatán & 2013 & 24,939 & 6.04207 & 302,857 & 190,849 \\
\hline Yucatán & 2014 & 26,052 & 6.23448 & 312,468 & 197,039 \\
\hline Yucatán & 2015 & 28,200 & 6.27426 & 324,908 & 204,021 \\
\hline Zacatecas & 2003 & 8,571 & 6.63978 & 101,987 & 92,835 \\
\hline Zacatecas & 2004 & 9,070 & 6.69120 & 103,305 & 96,594 \\
\hline Zacatecas & 2005 & 10,550 & 6.49772 & 104,547 & 95,111 \\
\hline Zacatecas & 2006 & 11,200 & 6.59094 & 106,978 & 100,909 \\
\hline Zacatecas & 2007 & 11,844 & 6.44558 & 113,491 & 104,091 \\
\hline Zacatecas & 2008 & 13,301 & 6.34683 & 121,691 & 113,082 \\
\hline Zacatecas & 2009 & 13,588 & 6.06205 & 124,912 & 116,924 \\
\hline Zacatecas & 2010 & 15,243 & 5.92325 & 131,692 & 129,488 \\
\hline Zacatecas & 2011 & 14,934 & 5.89561 & 137,704 & 129,942 \\
\hline Zacatecas & 2012 & 14,474 & 5.98326 & 143,177 & 134,255 \\
\hline Zacatecas & 2013 & 13,633 & 6.01694 & 146,735 & 133,225 \\
\hline Zacatecas & 2014 & 16,248 & 6.26694 & 153,739 & 140,820 \\
\hline Zacatecas & 2015 & 17,255 & 6.26258 & 161,165 & 141,972 \\
\hline
\end{tabular}




\section{Apéndice 2. Pruebas de Hausman}

Test de Hausman para discriminar entre efectos fijos y aleatorios. 32 entidades, 2003-2015

$\mathrm{H}_{0}$ : no hay correlación entre las variables independientes y los efectos aleatorios

\begin{tabular}{|l|r|r|r|}
\hline Resumen Test & Estadístico $\mathrm{Chi}^{2}$ & $\mathrm{Chi}^{2}$ d.f. & Prob. \\
\hline Cross-section random & 2.230432 & 3 & 0.5260 \\
\hline
\end{tabular}

* Para realizar esta prueba se omitió la variable CRISIS.

Fuente: Elaboración propia usando Eviews 7.2.

Tabla 2

Test de Hausman para discriminar entre efectos fijos y aleatorios. Frontera Norte, 2003-2015

\begin{tabular}{|l|c|r|c|}
\hline $\mathrm{H}_{0}$ : no hay correlación entre las variables independientes y los efectos aleatorios \\
\hline Resumen Test & Estadístico $\mathrm{Chi}^{2}$ & Chi $^{2}$ d.f. & Prob. \\
\hline Cross-section random & 0.000000 & 4 & 1.0000 \\
\hline
\end{tabular}

Fuente: Elaboración propia usando Eviews 7.2.

Tabla 3

Test de Hausman para discriminar entre efectos fijos y aleatorios. Sur, 2003-2015

$\mathrm{H}_{0}$ : no hay correlación entre las variables independientes y los efectos aleatorios

\begin{tabular}{|l|r|r|r|}
\hline Resumen Test & Estadístico $\mathrm{Chi}^{2}$ & Chi $^{2}$ d.f. & Prob. \\
\hline Cross-section random & 0.000000 & 4 & 1.0000 \\
\hline
\end{tabular}

Fuente: Elaboración propia usando Eviews 7.2.

Apéndice 3. Modelo con variables ficticias para las dos regiones de interés

Estimación con mínimos cuadrados ordinarios, 32 entidades, 2003-2015 (416 obs.)

\begin{tabular}{lrrl}
\hline \multicolumn{4}{l}{ Variable dependiente LOGLA } \\
\hline & \multicolumn{1}{l}{ Coeficiente } & Estadístico t & Prob. \\
\hline LOGLE & -0.239807 & -0.680590 & 0.4965 \\
LOGPIBNMAN & $0.644923^{* * *}$ & 22.28810 & 0.0000 \\
LOGPIBMAN & $0.232638^{* * *}$ & 12.50636 & 0.0000 \\
CRISIS & -0.010394 & -0.231118 & 0.8173 \\
SUR & $-0.344437^{* *}$ & -8.016666 & 0.0000 \\
FRONTERA & $0.157375^{* * *}$ & 3.401496 & 0.0007 \\
R $^{2}$ ajustado & 0.856758 & & \\
Estadístico F & 414.6995 & & \\
Prob. (Est.-F) & 0.000000 & & \\
\hline
\end{tabular}

$* * *$ Significativo al $99 \%$.

Fuente: Elaboración propia usando Eviews 7.2. 